\title{
Vekua Type Hierarchical Models for Prismatic Shells with Mixed Conditions on Face Surfaces
}

\author{
George Jaiani \\ Iv. Javakhishvili Tbilisi State University \\ Department of Mathematics $\mathcal{E}$ I. Vekua Institute of Applied Mathematics
}

\begin{abstract}
I.Vekua constructed hierarchical models for elastic prismatic shells, in particular, plates of variable thickness, when on the face surfaces either stresses or displacements are known. In the present paper other hierarchical models for cusped, in general, elastic isotropic and anisotropic prismatic shells are constructed and analyzed, namely, when on the face surfaces (i) a normal to the projection of the prismatic shell component of a stress vector and parallel to the projection of the prismatic shell components of a displacement vector, (ii) a normal to the projection of the prismatic shell component of the displacement vector and parallel to the projection of the prismatic shell components of the stress vector are prescribed. We construct also hierarchical models, when other mixed conditions are given on face surfaces. In the zero approximations of the models under consideration peculiarities (depending on sharpening geometry of the cusped edge) of correct setting boundary conditions at edges are investigated. In concrete cases some boundary value problems are solved in an explicit form. As an example of application of the constructed Vekua-type models to composite structures, as example, an unidirectional lamina with fibers parallel to $x_{2}$-axis under shear strain is considered. Tension-compression is treated as well.
\end{abstract}

\section{(c) 2011 Published by Elsevier Ltd.}

Keywords: Hierarchical models; elastic cusped prismatic shells; cusped prismatic shells; mixed conditions on face surfaces; composite structures

\section{Introduction}

Let $O x_{1} x_{2} x_{3}$ be an anticlockwise-oriented rectangular Cartesian frame of origin $O$. We conditionally assume the $x_{3}$-axis vertical. The elastic body is called a prismatic shell [1]-[3] if it is bounded above and below by, respectively, the surfaces (so called face surfaces)

$$
x_{3}=\stackrel{(+)}{h}\left(x_{1}, x_{2}\right) \text { and } x_{3}=\stackrel{(-)}{h}\left(x_{1}, x_{2}\right), \quad\left(x_{1}, x_{2}\right) \in \omega
$$

laterally by a cylindrical surface $\Gamma$ of generatrix parallel to the $x_{3}$-axis and its vertical dimension is sufficiently small compared with other dimensions of the body. $\bar{\omega}:=\omega \cup \partial \omega$ is the so-called projection of the prismatic shell on $x_{3}=0$.

Let the thickness of the prismatic shell be

$$
2 h\left(x_{1}, x_{2}\right):=\stackrel{(+)}{h}\left(x_{1}, x_{2}\right)-\stackrel{(-)}{h}\left(x_{1}, x_{2}\right) \begin{cases}>0 & \text { for } \quad\left(x_{1}, x_{2}\right) \in \omega \\ \geq 0 & \text { for } \quad\left(x_{1}, x_{2}\right) \in \partial \omega\end{cases}
$$


and

$$
\widetilde{2}\left(x_{1}, x_{2}\right):=\stackrel{(+)}{h}\left(x_{1}, x_{2}\right)+\stackrel{(-)}{h}\left(x_{1}, x_{2}\right) .
$$

If the thickness of the prismatic shell vanishes on some subset of $\partial \omega$, it is called cusped one.

Let us note that the lateral boundary of the standard shell is orthogonal to the "middle surface" of the shell, while the lateral boundary of the prismatic shell is orthogonal to the prismatic shell's projection on $x_{3}=0$.

I. Vekua [1], [2] constructed hierarchical models for elastic prismatic shells, in particular, plates of variable thickness, when on the face surfaces either stresses (Model I) or displacements (Model II) are known. He and his followers have investigated various aspects of the first model (for a survey see [3] and the introduction in [4]). The up-dated survey of results concerning cusped elastic prismatic shells in the cases of the first and second models is given in [3]. In the present paper six (Model III - Model VIII) hierarchical models for cusped, in general, elastic prismatic shells are constructed and analyzed, when on the face surfaces (i) a normal to the projection of the prismatic shell component $Q_{\frac{ \pm}{v} 3}$ of a stress vector and parallel to the projection of the prismatic shell components $u_{\alpha}\left(x_{1}, x_{2}, \stackrel{( \pm)}{h}, t\right), \alpha=1,2$, of a displacement vector (Model III), (ii) a normal to the projection of the prismatic shell component $u_{3}\left(x_{1}, x_{2}, \stackrel{( \pm)}{h, t}, t\right)$ of the displacement vector and parallel to the projection of the prismatic shell components $Q_{( \pm)}, \alpha=1,2$, of the stress vector (Model IV) are known. $\stackrel{(+)}{v}$ and $\stackrel{(-)}{v}$ denote outward normals to the face surfaces $\stackrel{(+)}{h}$ and $\stackrel{(-)}{h}$, respectively. Hierarchical Models we will call Model V and Model VI, when on the one face surface conditions (i) and on the other one conditions (ii) are prescribed. Besides we construct hierarchical models when on the upper face surface stress vector and on the lower face surface displacements (Model VII) and vice versa (Model VIII) are prescribed. In the zero approximations of the models under consideration peculiarities (depending on sharpening geometry of the cusped edge) of correct setting boundary conditions at edges are investigated. In concrete cases some boundary value problems are solved in an explicit form. In what follows $X_{i j}$ and $e_{i j}$ are the stress and strain tensors, respectively, $u_{i}$ are the displacements, $\Phi_{i}$ are the volume force components, $\rho$ is the density, $\lambda$ and $\mu$ are the Lamé constants, $\delta_{i j}$ is the Kronecker delta, subscripts preceded by a comma mean partial derivatives with respect to the corresponding variables. Moreover, repeated indices imply summation (Greek letters run from 1 to 2 and Latin letters run from 1 to 3 ).

Composite materials consist at least of two combined materials (see, e.g., [5], [6] and the references given there), commonly referred to as constituents. In general, one material is continuous, which is called the matrix, while other materials are discontinuous, mostly more stiff and are called reinforcements. The properties of a composite material depend on the properties of the constituents, of the geometry of the reinforcements, which usually is mesured by the volume fraction, and the nature and quality of the matrix-reinforcement interface. The most important aspect of composite materials in which the reinforcements are fibers is the anisotropy caused by the fiber orientation. But the properties of the fibres and the matrix can be smeared into an equivalent homogeneous material with orthotropic behaviour.

In the case of the general anisotropy the generalized Hooke's law looks like

$$
X_{i j}=E_{i j k l} e_{k l}, \quad i, j=1,2,3,
$$

where $E_{i j k l}$ are material coefficients ( 21 of them are independent). Orthotropic material behavior is typical for unidirectional laminae with on-axis loading. An orthotropic material has 12 non-zero and 9 independent material parameters $\left(E_{1111}, E_{1122}, E_{1133}, E_{2222}, E_{2233}, E_{3333}, E_{1212}, E_{2323}, E_{3131}\right)$. The stress-strain coupling is the same as for isotropic behavior: normal stresses $\left(X_{i i}, i=1,2,3\right)$ give rise to only extensional strains $\left(e_{i i}, i=1,2,3\right)$ and shear stresses $\left(X_{i j}, i \neq j, i, j=1,2,3\right)$ give rise to only shear strains $\left(e_{i j}, i \neq j, i, j=1,2,3\right)$ :

$$
\begin{gathered}
X_{i \underline{i}}=\sum_{k=1}^{3} E_{i \underline{i} k k} e_{k k}, \quad i=1,2,3 ; \\
X_{i j}=2 E_{\underline{i} \underline{j} i j} e_{i j}, \quad i \neq j, i, j=1,2,3 .
\end{gathered}
$$

A bar under one of repeated indices means that Einstein's summation convention is not used with respect to these indices. Otherwise we use this convention.

In most structural applications the structural elements are simplified models by reducing the three-dimensional state of stress and strain approximately to two-dimensional plane stress and plane strain state. A thin lamina (layer), 
for instance, can be considered to be under a condition of plane stress with all the stress components in the out-of-plane direction being zero, or its stress-strain state can be considered within the framework of different two-dimensional models (see, e.g., [7]-[9], and also [10]). It should be noted that in this spirit properly the Vekua hierarchical models [1]-[3] and its generalizations (see [11]-[13], [3] and the references given there) for non-linear, anisotropic and other type shells and plates as the theoretical backgrounds of engineering models are not duely appraised by engineers up to now. The main goal of the publication of the present paper in "Composite Structures" is twofold:

(i) to make readers (mostly engineers with a view to offer variety of refined models) be acquainted with the Vekua type two-dimensional hierarchical models according to which [14], [15] displacements $u_{i}, \quad i=1,2,3$, in $N:=\left(N_{1}, N_{2}, N_{3}\right), \quad N_{i}=0,1,2 \ldots, \quad i=1,2,3$, approximation are given in the following form (different choice of $N_{1}, N_{2}, N_{3}$ gives variety of models)

$$
u_{i}\left(x_{1}, x_{2}, x_{3}\right)=\sum_{n_{i}=0}^{N_{i}}\left(n_{i}+\frac{1}{2}\right) a\left(x_{1}, x_{2}\right) u_{\underline{i n} n_{i}}\left(x_{1}, x_{2}\right) P_{n_{i}}\left(a x_{3}-b\right), \quad i=1,2,3,
$$

where $P_{n_{i}}$ are the Legendre polynomials, $u_{i n_{i}}, \quad n_{i}=0,1, \ldots, N_{i}, \quad i=1,2,3$, are solutions of the corresponding two-dimensional governing systems (see [14], [15]),

$$
a\left(x_{1}, x_{2}\right):=\frac{1}{h\left(x_{1}, x_{2}\right)}, \quad b\left(x_{1}, x_{2}\right):=\frac{\stackrel{(+)}{h}\left(x_{1}, x_{2}\right)+\stackrel{(-)}{h}\left(x_{1}, x_{2}\right)}{2 h\left(x_{1}, x_{2}\right)},
$$

$2 h\left(x_{1}, x_{2}\right):=\stackrel{(+)}{h}\left(x_{1}, x_{2}\right)-\stackrel{(-)}{h}\left(x_{1}, x_{2}\right)$ is a thickness of the prismatic shell [1-3] (in the case of plates of variable thickness $\left.b\left(x_{1}, x_{2}\right) \equiv 0\right)$

(ii) to explain peculiarities of setting boundary conditions at the cusped edges (i.e. the thickness is vanishing at the edge) of the matrix.

In the present paper, for the readers convenience, we construct only $(0,0,0)$ hierarchical models, in general, with mixed conditions on the face surfaces, on the basis of linear theory of isotropic elastic materials. The cases of the orthotropic and general anisotropic materials can be handle in the same way; for clearness we do it for the case of Model III. The mechanical behavior of a composite material in the sense of the proportion of the matrix and of the reinforcement, and the material characteristics can be expressed by their volume or weight fraction in an usual manner. Their volume fractions usually lies between 0.3 and 0.7 [6]. In order to illustrate an application of Vekua-type models to composite structures, as example, an unidirectional lamina with fibers parallel to $x_{2}$-axis under shear strain is considered. Tension-compression is treated as well (see Remark 1 and Section 4).

According to I.Vekua's [1, 2] dimension reduction method, in order to construct hierarchical models for elastic prismatic shells we multiply the basic equations of linear three-dimensional elasticity,

Motion Equations

$$
X_{i j, j}+\Phi_{i}=\rho \frac{\partial^{2} u_{i}}{\partial t^{2}}\left(x_{1}, x_{2}, x_{3}, t\right), \quad\left(x_{1}, x_{2}, x_{3}\right) \in \Omega \subset \mathbb{R}^{3}, \quad t>t_{0}, \quad i=1,2,3 ;
$$

\section{Generalized Hooke's law (isotropic case)}

$$
X_{i j}=\lambda \theta \delta_{i j}+2 \mu e_{i j}, \quad i, j=1,2,3, \quad \theta:=e_{i i}
$$

\section{Kinematic Relations}

$$
e_{i j}=\frac{1}{2}\left(u_{i, j}+u_{j, i}\right), \quad i, j=1,2,3
$$

by Legendre polynomials $P_{l}\left(a x_{3}-b\right)$ and then we integrate with respect to the thickness variable $x_{3}$ within the limits $\stackrel{(-)}{h}\left(x_{1}, x_{2}\right)$ and $\stackrel{(-)}{h}\left(x_{1}, x_{2}\right)$. By these calculations prescribed on upper and lower face surfaces some components of the stress and displacement vectors are assumed as known, while the values of other components on the face surfaces are calculated from their Fourier-Legendre series expansions on the segment $x_{3} \in\left[\stackrel{(-)}{h}\left(x_{1}, x_{2}\right), \stackrel{(+)}{h}\left(x_{1}, x_{2}\right)\right]$. So, we get the 
equivalent infinite system of relations with respect to the so called $l$-th order moments

$$
\begin{array}{r}
\left(X_{i j l}, e_{i j l}, u_{i l}\right)\left(x_{1}, x_{2}, t\right):=\int_{(-)}^{\stackrel{(+)}{h}}\left(X_{i j}, e_{i j}, u_{i}\right)\left(x_{1}, x_{2}, x_{3}, t\right) P_{l}\left(a x_{3}-b\right) d x_{3}, \\
i, j=1,2,3, \quad l=0,1, \cdots .
\end{array}
$$

Then, having followed the usual procedure used in the theory of elasticity, we get an equivalent infinite system, consisting of three groups corresponding to each $i=1,2,3$ (to this end, see (1)), with respect to the $l$-th order moments $u_{i l}$. After this if we assume that the moments $u_{\underline{i n}}$ whose subscripts $n_{i}$, indicating moments' order, are greater than $N_{i}$, equal zero and consider for each $i=1,2,3$ only the first $N_{i}+1$ equations in the obtained infinite system of equations with respect to the $n_{i}$-th order moments $u_{i n_{i}}$ we obtain the $N=\left(N_{1}, N_{2}, N_{3}\right)$ approximation (hierarchical model) governing system with respect to $u_{\underline{i n} i_{i}}$ or

$$
\stackrel{N}{v}_{\underline{i n}_{i}}:=\frac{\stackrel{N}{\underline{i n}}_{i}}{h^{n_{i}+1}}, \quad i=1,2,3, \quad n_{i}=\overline{0, N_{i}}
$$

(roughly speaking ${ }_{i n_{i}}^{N}$ is an "approximate value" of $u_{i n_{i}}$ ). Similarly, we treat the case of the general anisotropic, in particular, orthotropic non-homogeneous materials.

\section{Construction of Models}

For the sake of transparency we confine ourselves to immediate deriving the $N=(0,0,0)$ approximation. To this end, we will need only integration of the basic $3 D$ relations of the theory of elasticity keeping in mind quantities prescribed on the face surfaces.

Integrating basic equations (1)-(3) with respect to $x_{3}$ from $\stackrel{(-)}{h}\left(x_{1}, x_{2}\right)$ to $\stackrel{(+)}{h}\left(x_{1}, x_{2}\right)$ and, taking into account (4), we get

$$
\begin{aligned}
& X_{j \beta 0, \beta}\left(x_{1}, x_{2}, t\right)-X_{j \beta}\left(x_{1}, x_{2}, \stackrel{(+)}{h}\left(x_{1}, x_{2}\right), t\right) \stackrel{(+)}{h}, \beta \\
& +X_{j 3}\left(x_{1}, x_{2}, \stackrel{(+)}{h}\left(x_{1}, x_{2}\right), t\right)+X_{j \beta}\left(x_{1}, x_{2}, \stackrel{(-)}{h}\left(x_{1}, x_{2}\right), t\right) \stackrel{(-)}{h}, \beta \\
& -X_{j 3}\left(x_{1}, x_{2}, \stackrel{(-)}{h}\left(x_{1}, x_{2}\right), t\right)+\Phi_{j 0}\left(x_{1}, x_{2}, t\right)=\rho \frac{\partial^{2} u_{j 0}\left(x_{1}, x_{2}, t\right)}{\partial t^{2}}, j=1,2,3 \text {; } \\
& X_{i j 0}\left(x_{1}, x_{2}, t\right)=\lambda e_{k k 0}\left(x_{1}, x_{2}, t\right) \delta_{i j}+2 \mu e_{i j 0}\left(x_{1}, x_{2}, t\right), \quad i, j=1,2,3 \\
& e_{i \beta 0}\left(x_{1}, x_{2}, t\right)=\frac{1}{2}\left[u_{i 0, \beta}\left(x_{1}, x_{2}, t\right)+u_{i}\left(x_{1}, x_{2}, \stackrel{(-)}{h}, t\right) \stackrel{(-)}{h}, \beta, u_{i}\left(x_{1}, x_{2}, \stackrel{(+)}{h}, t\right) \stackrel{(+)}{h}, \beta\right. \\
& +\left\{\begin{array}{l}
u_{\beta 0, \alpha}\left(x_{1}, x_{2}, t\right)-u_{\beta}\left(x_{1}, x_{2}, \stackrel{(+)}{h}, t\right) \stackrel{(+)}{h},{ }_{\alpha}+u_{\beta}\left(x_{1}, x_{2}, \stackrel{(-)}{h}, t\right) \stackrel{(-)}{h}, \alpha, \quad i=\alpha, \\
u_{\beta}\left(x_{1}, x_{2}, \stackrel{h}{h}, t\right)-u_{\beta}\left(x_{1}, x_{2}, \stackrel{i}{h}, t\right), \quad i=3
\end{array}\right], \\
& i=1,2,3, \beta=1,2 \text {, }
\end{aligned}
$$

Model III. On the face surfaces

$$
\begin{aligned}
& Q_{v^{\prime} 3}\left(x_{1}, x_{2}, \stackrel{( \pm)}{h}\left(x_{1}, x_{2}\right), t\right)=X_{3 \beta}\left(x_{1}, x_{2}, \stackrel{( \pm)}{h}\left(x_{1}, x_{2}\right), t\right) \stackrel{( \pm)}{v}_{\beta}+X_{33}\left(x_{1}, x_{2}, \stackrel{( \pm)}{h}\left(x_{1}, x_{2}\right), t\right) \stackrel{( \pm)}{v_{3}}{ }_{3}, \\
& u_{\alpha}\left(x_{1}, x_{2}, \stackrel{( \pm)}{h}\left(x_{1}, x_{2}\right), t\right), \quad \alpha=1,2 \text {, }
\end{aligned}
$$


are known.

Model IV.On the face surfaces

$$
\begin{aligned}
Q_{( \pm \alpha}\left(x_{1}, x_{2}, \stackrel{( \pm)}{h}\left(x_{1}, x_{2}\right), t\right) & =X_{\alpha \beta}\left(x_{1}, x_{2}, \stackrel{( \pm)}{h}\left(x_{1}, x_{2}\right), t\right) \stackrel{( \pm)}{v}_{\beta}+X_{\alpha 3}\left(x_{1}, x_{2}, \stackrel{( \pm)}{h}\left(x_{1}, x_{2}\right), t\right) \stackrel{( \pm)}{v_{3}}, \\
& u_{3}\left(x_{1}, x_{2}, \stackrel{( \pm)}{h}\left(x_{1}, x_{2}\right), t\right), \quad \alpha=1,2,
\end{aligned}
$$

are known.

Model V.

$$
\begin{aligned}
& \text { On the upper surface } \stackrel{(+)}{h} \text { the quantities (10) are known, } \\
& \text { on the lower surface } \stackrel{(-)}{h} \text { the quantities (11) are known. }
\end{aligned}
$$

(+) $(-)$

Model VI. On $\stackrel{(+)}{h}$ the quantities (11) and on $\stackrel{(-)}{h}$ the quantities (10) are known.

Model VII. On the upper face surface stress vector components $Q_{v i}^{(+)}, i=1,2,3$, and on the lower face surface displacements are known.

Model VIII.On the upper face surface displacements and on the lower face surface stress vector components $Q_{\substack{(-) \\ i}}$, $i=1,2,3$, are known.

In the $N=0$ approximation it is assumed that

$$
\begin{gathered}
u_{i}\left(x_{1}, x_{2}, x_{3}, t\right) \cong \frac{u_{i 0}\left(x_{1}, x_{2}, t\right)}{2 h}=: \frac{1}{2} v_{i 0}\left(x_{1}, x_{2}, t\right), \quad i=1,2,3, \\
X_{i j}\left(x_{1}, x_{2}, x_{3}, t\right) \cong \frac{X_{i j 0}\left(x_{1}, x_{2}, t\right)}{2 h}, \quad i=1,2,3,
\end{gathered}
$$

In the case of Model III, taking into account (14), (15), from (6), (9), (8) we obtain correspondingly

$$
\begin{aligned}
& X_{3 \beta 0, \beta}+Q_{\stackrel{(+)}{3}_{3}} \sqrt{(\stackrel{(+)}{h}, 1)^{2}+(\stackrel{(+)}{h}, 2)^{2}+1}+Q_{\stackrel{(-)}{3}^{-}} \sqrt{(\stackrel{(-)}{h}, 1))^{2}+(\stackrel{(-)}{h}, 2)^{2}+1}+\Phi_{30}=\rho \frac{\partial^{2} u_{30}}{\partial t^{2}} \\
& X_{\alpha \beta 0, \beta}-\frac{1}{2 h}\left[X_{\alpha \beta 0}\left(\stackrel{(+)}{h}, \beta_{, \beta} \stackrel{(-)}{h}, \beta\right)-X_{\alpha 30}+X_{\alpha 30}\right]+\Phi_{\alpha 0}=\rho \frac{\partial^{2} u_{\alpha 0}}{\partial t^{2}}, \quad \alpha=1,2,
\end{aligned}
$$

i.e.,

$$
\begin{aligned}
& X_{\alpha \beta 0, \beta}-(\ln h)_{, \beta} X_{\alpha \beta 0}+\Phi_{\alpha 0}=\rho \frac{\partial^{2} u_{\alpha 0}}{\partial t^{2}}, \quad \alpha=1,2 ; \\
& e_{330}=u_{3}\left(x_{1}, x_{2}, \stackrel{(+)}{h}, t\right)-u_{3}\left(x_{1}, x_{2}, \stackrel{(-)}{h}, t\right)=: \Psi_{33}=\frac{1}{2} v_{30}\left(x_{1}, x_{2}, t\right)-\frac{1}{2} v_{30}\left(x_{1}, x_{2}, t\right)=0, \\
& e_{3 \beta 0}=\frac{1}{2}\left\{u_{30, \beta}+\left[u_{\beta}\left(x_{1}, x_{2}, \stackrel{(+)}{h}, t\right)-u_{\beta}\left(x_{1}, x_{2}, \stackrel{(-)}{h}, t\right)\right]+\frac{1}{2} v_{30}(\stackrel{(-)}{h}, \stackrel{(+)}{h}, \stackrel{+}{h}, \beta)\right\} \\
& =\frac{1}{2}\left[\left(h v_{30}\right)_{, \beta}-h,_{\beta} v_{30}\right]+\frac{1}{2}\left[u_{\beta}\left(x_{1}, x_{2}, \stackrel{(+)}{h}, t\right)-u_{\beta}\left(x_{1}, x_{2}, \stackrel{(-)}{h}, t\right)\right] \\
& =\frac{1}{2} h v_{30, \beta}+\frac{1}{2}\left[u_{\beta}\left(x_{1}, x_{2}, \stackrel{(+)}{h}, t\right)-u_{\beta}\left(x_{1}, x_{2}, \stackrel{(-)}{h}, t\right)\right], \quad \beta=1,2 \text {; } \\
& e_{\alpha \beta 0}=\frac{1}{2}\left[u_{\alpha 0, \beta}+u_{\beta 0, \alpha}-u_{\beta}\left(x_{1}, x_{2}, \stackrel{(+)}{h}, t\right) \stackrel{(+)}{h},_{\alpha}+u_{\beta}\left(x_{1}, x_{2}, \stackrel{(-)}{h}, t\right) \stackrel{(-)}{h},_{\alpha}\right. \\
& \left.+u_{\alpha}\left(x_{1}, x_{2}, \stackrel{(-)}{h}, t\right) \stackrel{(-)}{h},_{, \beta}-u_{\alpha}\left(x_{1}, x_{2}, \stackrel{(+)}{h}, t\right) \stackrel{(+)}{h}, \beta\right]=\frac{1}{2}\left(u_{\alpha 0, \beta}+u_{\beta 0, \alpha}\right)+\Psi_{\alpha \beta} \\
& =\frac{1}{2}\left[\left(h v_{\alpha 0}\right)_{, \beta}+\left(h v_{\beta 0}\right)_{, \alpha}\right]+\Psi_{\alpha \beta}, \quad \alpha, \beta=1,2,
\end{aligned}
$$


where

$$
\begin{aligned}
\Psi_{\alpha \beta} & :=\frac{1}{2}\left[u_{\beta}\left(x_{1}, x_{2}, \stackrel{(-)}{h}, t\right) \stackrel{(-)}{h},,_{\alpha}-u_{\beta}\left(x_{1}, x_{2}, \stackrel{(+)}{h}, t\right) \stackrel{(+)}{h}, \alpha\right. \\
& \left.+u_{\alpha}\left(x_{1}, x_{2}, \stackrel{(-)}{h}, t\right) \stackrel{(-)}{h},_{\beta}-u_{\alpha}\left(x_{1}, x_{2}, \stackrel{(+)}{h}, t\right) \stackrel{(+)}{h}, \beta\right]
\end{aligned}
$$

Substituting (18)-(20) into (7), we get

$$
\begin{gathered}
X_{330}\left(x_{1}, x_{2}, t\right)=\lambda\left(u_{\gamma 0, \gamma}+\Psi_{\gamma \gamma}\right)=\lambda\left(h v_{\gamma 0}\right)_{\gamma}+\lambda \Psi_{\gamma \gamma}, \\
X_{3 \beta 0}\left(x_{1}, x_{2}, t\right)=\mu h v_{30, \beta}+\mu\left[u_{\beta}\left(x_{1}, x_{2}, \stackrel{(+)}{h}, t\right)-u_{\beta}\left(x_{1}, x_{2}, \stackrel{(-)}{h}, t\right)\right], \beta=1,2, \\
X_{\alpha \beta 0}\left(x_{1}, x_{2}, t\right)=\lambda\left(h v_{\gamma 0}\right)_{, \gamma} \delta_{\alpha \beta}+\lambda \Psi_{\gamma \gamma} \delta_{\alpha \beta}+\mu\left[\left(h v_{\alpha 0}\right)_{, \beta}+\left(h v_{\beta 0}\right)_{, \alpha}\right]+2 \mu \Psi_{\alpha \beta}, \alpha, \beta=1,2 .
\end{gathered}
$$

So, when normal to the projection of the prismatic shell components $Q_{(+)}$and $Q_{(-)}$of a stress vector and parallel to the projection of the prismatic shell components $u_{\alpha}\left(x_{1}, x_{2}, \stackrel{(+)}{h}, t\right)$ and $u_{\alpha}\left(x_{1}, x_{2}, \stackrel{(-)}{h}, t\right), \alpha=1,2$, of a displacement vector are known on $\stackrel{(+)}{h}$ and $\stackrel{(-)}{h}$, respectively, substituting (21)-(23) into (17), (16) the governing system for the weighted zero moments $v_{j 0}, j=1,2,3$, (see (5), superscript $N=(0,0,0)$ is omitted below) has the following form

$$
\begin{aligned}
& \mu\left(h v_{\alpha 0}\right)_{, \beta \beta}+(\lambda+\mu)\left(h v_{\gamma 0}\right)_{\gamma_{\alpha}}-(\ln h)_{, \beta}\left\{\lambda \delta_{\alpha \beta}\left(h v_{\gamma 0}\right)_{, \gamma}+\mu\left[\left(h v_{\alpha 0}\right)_{, \beta}+\left(h v_{\beta 0}\right)_{, \alpha}\right]\right\} \\
& +2 \mu \Psi_{\alpha \beta, \beta}+\lambda \Psi_{\gamma \gamma, \alpha}-(\ln h)_{, \beta}\left[\lambda \delta_{\alpha \beta} \Psi_{\gamma \gamma}+2 \mu \Psi_{\alpha \beta}\right]+\Phi_{\alpha 0}=\rho h \frac{\partial^{2} v_{\alpha 0}}{\partial t^{2}}, \quad \alpha=1,2 ; \\
& \mu\left(h v_{30, \beta}\right)_{, \beta}+\mu\left[u_{\beta}\left(x_{1}, x_{2}, \stackrel{(+)}{h}, t\right)-u_{\beta}\left(x_{1}, x_{2}, \stackrel{(-)}{h}, t\right)\right]_{, \beta}+Q_{v_{v 3}} \sqrt{(\stackrel{(+)}{h}, 1)^{2}+(\stackrel{(+)}{h}, 2)^{2}+1} \\
& +Q_{(-)} \sqrt{\left(\stackrel{(-)}{h},{ }_{1}\right)^{2}+(\stackrel{(-)}{h}, 2)^{2}+1}+\Phi_{30}=\rho h \frac{\partial^{2} v_{30}}{\partial t^{2}} .
\end{aligned}
$$

In this model $\Psi_{33} \equiv 0$ in contrast to the model, when on the face surfaces only displacements are prescribed (Model II).

In Model II $\Psi_{33} \not \equiv 0$, in general (see system (65) below).

Remark 1 In the case of the general anisotropic non-homogeneous materials formulas (7), (21)-(23) should be replaced by

$$
X_{i j 0}\left(x_{1}, x_{2}, t\right)=E_{i j k l}\left(x_{1}, x_{2}\right) e_{k l 0}\left(x_{1}, x_{2}, t\right)
$$

$$
\begin{aligned}
& X_{330}\left(x_{1}, x_{2}, t\right)=E_{33 \alpha \beta}\left(x_{1}, x_{2}\right) e_{\alpha \beta 0}\left(x_{1}, x_{2}, t\right)+2 E_{333 \beta}\left(x_{1}, x_{2}\right) e_{3 \beta 0}\left(x_{1}, x_{2}, t\right) \\
& =\frac{1}{2} E_{33 \alpha \beta}\left[\left(h v_{\alpha 0}\right)_{, \beta}+\left(h v_{\beta 0}\right)_{, \alpha}+2 \Psi_{\alpha \beta}\right]+E_{333 \beta}\left[h v_{30, \beta}+u_{\beta}\left(x_{1}, x_{2}, \stackrel{(+)}{h}, t\right)-u_{\beta}\left(x_{1}, x_{2}, \stackrel{(-)}{h}, t\right)\right] \text {, } \\
& X_{3 \delta 0}\left(x_{1}, x_{2}, t\right)=E_{3 \delta \alpha \beta}\left(x_{1}, x_{2}\right) e_{\alpha \beta 0}\left(x_{1}, x_{2}, t\right)+2 E_{3 \delta 3 \beta}\left(x_{1}, x_{2}\right) e_{3 \beta 0}\left(x_{1}, x_{2}, t\right) \\
& =\frac{1}{2} E_{3 \delta \alpha \beta}\left[\left(h v_{\alpha 0}\right)_{, \beta}+\left(h v_{\beta 0}\right)_{, \alpha}+2 \Psi_{\alpha \beta}\right] \\
& +E_{3 \delta 3 \beta}\left[h v_{30, \beta}+u_{\beta}\left(x_{1}, x_{2}, \stackrel{(+)}{h}, t\right)-u_{\beta}\left(x_{1}, x_{2}, \stackrel{(-)}{h}, t\right)\right], \quad \delta=1,2, \\
& X_{\gamma \delta 0}\left(x_{1}, x_{2}, t\right)=E_{\gamma \delta \alpha \beta}\left(x_{1}, x_{2}\right) e_{\alpha \beta 0}\left(x_{1}, x_{2}, t\right)+2 E_{\gamma \delta 3 \beta}\left(x_{1}, x_{2}\right) e_{3 \beta 0}\left(x_{1}, x_{2}, t\right) \\
& =\frac{1}{2} E_{\gamma \delta \alpha \beta}\left[\left(h v_{\alpha 0}\right)_{, \beta}+\left(h v_{\beta 0}\right)_{, \alpha}+2 \Psi_{\alpha \beta}\right] \\
& +E_{\gamma \delta 3 \beta}\left[h v_{30, \beta}+u_{\beta}\left(x_{1}, x_{2}, \stackrel{(+)}{h}, t\right)-u_{\beta}\left(x_{1}, x_{2}, \stackrel{(-)}{h}, t\right)\right], \quad \gamma, \delta=1,2,
\end{aligned}
$$


respectively. Substituting (29), (28) into (17), (16), we get the following governing equations:

$$
\begin{aligned}
& \frac{1}{2}\left\{E_{\gamma \delta \alpha \beta}\left[\left(h v_{\alpha 0}\right)_{, \beta}+\left(h v_{\beta 0}\right)_{, \alpha}\right]\right\}_{, \delta}+\left(E_{\gamma \delta 3 \beta} h v_{30, \beta}\right)_{, \delta} \\
& -\frac{1}{2}(\ln h)_{, \delta}\left\{E_{\gamma \delta \alpha \beta}\left[\left(h v_{\alpha 0}\right)_{, \beta}+\left(h v_{\beta 0}\right)_{, \alpha}\right]+2 E_{\gamma \delta 3 \beta} h v_{30, \beta}\right\} \\
& +\left(E_{\gamma \delta \alpha \beta} \Psi_{\alpha \beta}\right)_{, \delta}+\left\{E_{\gamma \delta 3 \beta}\left[u_{\beta}\left(x_{1}, x_{2}, \stackrel{(+)}{h}, t\right)-u_{\beta}\left(x_{1}, x_{2}, \stackrel{(-)}{h}, t\right)\right]\right\}_{, \delta} \\
& \left.-(\ln h), \delta\left\{E_{\gamma \delta \alpha \beta} \Psi_{\alpha \beta}+E_{\gamma \delta 3 \beta}\left[u_{\beta}\left(x_{1}, x_{2}, h^{(+)}, t\right)-u_{\beta} x_{1}, x_{2}, h^{(-)}, t\right)\right]\right\} \\
& +\Phi_{\gamma 0}=\rho h \frac{\partial^{2} v_{\gamma 0}}{\partial t^{2}}, \quad \gamma=1,2 \\
& \frac{1}{2}\left\{E_{3 \delta \alpha \beta}\left[\left(h v_{\alpha 0, \beta}\right)+\left(h v_{\beta 0}\right)_{, \alpha}\right], \delta+\left(E_{3 \delta 3 \beta} h v_{30, \beta}\right), \delta\right\} \\
& +\left(E_{3 \delta \alpha \beta} \Psi_{\alpha \beta}\right)_{, \delta}+\left\{E_{3 \delta 3 \beta}\left[u_{\beta}\left(x_{1}, x_{2}, \stackrel{(+)}{h}, t\right)-u_{\beta}\left(x_{1}, x_{2}, \stackrel{(-)}{h}, t\right)\right]\right\}_{, \delta} \\
& +Q_{v 3} \sqrt{(\stackrel{(+)}{h}, 1)^{2}+(\stackrel{(+)}{h}, 2)^{2}+1}+Q_{v^{(-)} 3} \sqrt{(\stackrel{(-)}{h}, 1)^{2}+(\stackrel{(-)}{h}, 2)^{2}+1} \\
& +\Phi_{30}=\rho h \frac{\partial^{2} v_{30}}{\partial t^{2}} \text {. }
\end{aligned}
$$

In the case of an orthropic material the governing system has the following form

$$
\begin{aligned}
& \frac{1}{2}\left\{\delta_{\gamma \alpha} \delta_{\alpha \beta} E_{\gamma \delta \alpha \beta}\left[\left(h v_{\alpha 0}\right)_{, \beta}+\left(h v_{\beta 0}\right), \alpha\right]\right\}_{, \delta} \\
& -\frac{1}{2}(\ln h)_{, \delta} \delta_{\gamma \delta} \delta_{\alpha \beta} E_{\gamma \delta \alpha \beta}\left[\left(h v_{\alpha 0}\right)_{, \beta}+\left(h v_{\beta 0}\right)_{, \alpha}\right] \\
& +\left(\delta_{\gamma \delta} \delta_{\alpha \beta} \Psi_{\alpha \beta}\right)_{, \delta}-(\ln h), \delta \delta_{\gamma \delta} \delta_{\alpha \beta} \Psi_{\alpha \beta}+\Phi_{\gamma 0}=\rho h \frac{\partial^{2} v_{\gamma 0}}{\partial t^{2}}, \quad \gamma=1,2 ; \\
& \frac{1}{2}\left(\delta_{\delta \beta} E_{3 \delta 3 \beta} h v_{30, \beta}\right)_{, \delta}+\left\{\delta_{\delta \beta} e_{3 \delta 3 \beta}\left[u_{\beta}\left(x_{1}, x_{2}, \stackrel{(+)}{h, t}\right)-u_{\beta}\left(x_{1}, x_{2}, \stackrel{(-)}{h}, t\right)\right]\right\}_{, \delta} \\
& +Q_{(+)} \sqrt{(\stackrel{(+)}{h}, 1)^{2}+(\stackrel{(+)}{h}, 2)^{2}+1}+Q_{v^{(-) 3}} \sqrt{(\stackrel{(-)}{h}, 1)^{2}+(\stackrel{(-)}{h}, 2)^{2}+1} \\
& +\Phi_{30}=\rho h \frac{\partial^{2} v_{30}}{\partial t^{2}} .
\end{aligned}
$$

As we see the last system is split into an equation for $v_{30}$ and a separate system for $v_{\alpha 0}, \quad \alpha=1,2$, like the case of an isotropic material.

Now, we consider Model IV. In the case of Model IV taking into account (14), (15) from (6), (9), (8) we have correspondingly

$$
\begin{array}{r}
X_{\alpha \beta 0, \beta}+{\underset{v \alpha \alpha}{Q_{(+)}}} \sqrt{(\stackrel{(+)}{h}, 1)^{2}+(\stackrel{(+)}{h}, 2)^{2}+1}+Q_{\left(_{\nu \alpha}\right)} \sqrt{(\stackrel{(-)}{h}, 1)^{2}+(\stackrel{(-)}{h}, 2)^{2}+1}+\Phi_{\alpha 0} \\
=\rho h \frac{\partial^{2} v_{\alpha 0}}{\partial t^{2}}, \quad \alpha=1,2, \\
X_{3 \beta 0, \beta}-\frac{1}{2 h}\left[X_{3 \beta 0}(\stackrel{(+)}{h}, \stackrel{(-)}{h}, \beta)-X_{330}+X_{330}\right]+\Phi_{30}=\rho h \frac{\partial^{2} v_{30}}{\partial t^{2}},
\end{array}
$$


i.e.,

$$
\begin{aligned}
& X_{3 \beta 0, \beta}-(\ln h)_{, \beta} X_{3 \beta 0}+\Phi_{30}=\rho h \frac{\partial^{2} v_{30}}{\partial t^{2}} ; \\
& e_{330}=u_{3}\left(x_{1}, x_{2}, \stackrel{(+)}{h}, t\right)-u_{3}\left(x_{1}, x_{2}, \stackrel{(-)}{h}, t\right)=: \Psi_{33}, \\
& e_{3 \beta 0}=\frac{1}{2}\left[u_{30, \beta}+u_{\beta}\left(x_{1}, x_{2}, \stackrel{(+)}{h}, t\right)-u_{\beta}\left(x_{1}, x_{2}, \stackrel{(-)}{h}, t\right)+u_{3}\left(x_{1}, x_{2}, \stackrel{(-)}{h}, t\right) \stackrel{(-)}{h}{ }_{\beta}-u_{3}\left(x_{1}, x_{2}, \stackrel{(+)}{h}, t\right)^{(+)}{ }_{, \beta}\right] \\
& =\frac{1}{2}\left[\left(h v_{30}\right)_{, \beta}+\frac{u_{\beta 0}\left(x_{1}, x_{2}, t\right)}{2 h}-\frac{u_{\beta 0}\left(x_{1}, x_{2}, t\right)}{2 h}+u_{3}\left(x_{1}, x_{2}, \stackrel{(-)}{h}, t\right) \stackrel{(-)}{h}{ }_{\beta}-u_{3}\left(x_{1}, x_{2}, \stackrel{(+)}{h}, t\right) \stackrel{(+)}{h},{ }_{\beta}\right] \\
& =\frac{1}{2}\left[\left(h v_{30}\right)_{\beta}+u_{3}\left(x_{1}, x_{2}, \stackrel{(-)}{h}, t\right) \stackrel{(-)}{h}{ }_{\beta}-u_{3}\left(x_{1}, x_{2}, \stackrel{(+)}{h}, t\right) \stackrel{(+)}{h}_{, \beta}\right], \beta=1,2, \\
& e_{\alpha \beta 0}=\frac{1}{2}\left[u_{\alpha 0, \beta}+u_{\beta 0, \alpha}-u_{\beta}\left(x_{1}, x_{2}, \stackrel{(+)}{h}, t\right) \stackrel{(+)}{h}{ }_{, \alpha}+u_{\beta}\left(x_{1}, x_{2}, \stackrel{(-)}{h}, t\right) \stackrel{(-)}{h}, \alpha\right. \\
& \left.+u_{\alpha}\left(x_{1}, x_{2}, \stackrel{(-)}{h}, t\right) \stackrel{(-)}{h}{ }_{, \beta}-u_{\alpha}\left(x_{1}, x_{2}, \stackrel{(+)}{h}, t\right) \stackrel{(+)}{h},{ }_{\beta}\right] \\
& \left.=\frac{1}{2}\left[\left(h v_{\alpha 0}\right)_{, \beta}+\left(h v_{\beta 0}\right)_{, \alpha}-v_{\beta 0} h_{, \alpha}-v_{\alpha 0} h_{, \beta}\right]=\frac{1}{2} h\left(v_{\alpha 0, \beta}+v_{\beta 0}\right)_{, \alpha}\right), \quad \alpha=1,2 .
\end{aligned}
$$

Substituting (36)-(38) into (7), we get

$$
\begin{gathered}
X_{330}=\lambda\left[h v_{\gamma 0, \gamma}+\Psi_{33}\right]+2 \mu \Psi_{33}, \\
X_{3 \beta 0}=\mu\left[\left(h v_{30}\right)_{, \beta}+u_{3}\left(x_{1}, x_{2}, \stackrel{(-)}{h}, t\right) \stackrel{(-)}{h},_{, \beta}-u_{3}\left(x_{1}, x_{2}, \stackrel{(+)}{h}, t\right) \stackrel{(+)}{h}, \beta\right], \beta=1,2, \\
X_{\alpha \beta 0}=\lambda\left[h v_{\gamma 0, \gamma}+\Psi_{33}\right] \delta_{\alpha \beta}+\mu h\left(v_{\beta 0, \alpha}+v_{\alpha 0, \beta}\right), \quad \alpha, \beta=1,2 .
\end{gathered}
$$

So, when normal to the projection of the prismatic shell components $u_{3}\left(x_{1}, x_{2}, \stackrel{(+)}{h}, t\right)$ and $u_{3}\left(x_{1}, x_{2}, \stackrel{(-)}{h}, t\right)$ of the displacement vector and parallel to the projection of the prismatic shell components $Q_{(+) \alpha}$ and $Q_{(\cdot) \alpha}, \alpha=1,2$, of the stress vector are prescribed on $\stackrel{(+)}{h}$ and $\stackrel{(-)}{h}$, respectively, substituting (39)-(41) into (34), (35) the governing system for the weighted zero moments $v_{j 0}, j=1,2,3$, has the following form

$$
\begin{aligned}
& \mu\left(h v_{\alpha 0, \beta}\right)_{, \beta}+\mu\left(h v_{\beta 0, \alpha}\right)_{, \beta}+\lambda\left(h v_{\gamma 0, \gamma}\right)_{, \alpha}+\lambda \Psi_{33, \alpha}+Q_{v_{\nu \alpha}} \sqrt{(\stackrel{(+)}{h, 1})^{2}+(\stackrel{(+)}{h, 2})^{2}+1} \\
& +Q_{(-)} \sqrt{(\stackrel{(-)}{h}, 1)^{2}+(\stackrel{(-)}{h, 2})^{2}+1}+\Phi_{\alpha 0}=\rho h \frac{\partial^{2} v_{30}}{\partial t^{2}}, \quad \alpha=1,2, \\
& \mu\left(h v_{30}\right)_{\beta \beta}-\mu(\ln h)_{, \beta}\left(h v_{30}\right)_{\beta}+\mu\left[u_{3}\left(x_{1}, x_{2}, \stackrel{(-)}{h}, t\right) \stackrel{(-)}{h}{ }_{, \beta}-u_{3}\left(x_{1}, x_{2}, \stackrel{(+)}{h}, t\right)_{h_{\beta}}^{(+)}\right]_{, \beta} \\
& -\mu(\ln h)_{, \beta}\left[u_{3}\left(x_{1}, x_{2}, \stackrel{(-)}{h}, t\right)_{(-)}^{h}{ }_{, \beta}-u_{3}\left(x_{1}, x_{2}, \stackrel{(+)}{h}, t\right) \stackrel{(+)}{h}_{, \beta}\right]+\Phi_{30}=\rho h \frac{\partial^{2} v_{30}}{\partial t^{2}} .
\end{aligned}
$$

In this model $\Psi_{33} \neq \equiv 0$, in general (it is identically zero if $u_{3}\left(x_{1}, x_{2}, \stackrel{(+)}{h}, t\right)=u_{3}\left(x_{1}, x_{2}, \stackrel{(-)}{h}, t\right)$ ), in contrast to the Model I (see system (63) below), when on the face surfaces only stress vectors are known (in the last case $\Psi_{33} \equiv 0$ ). In the similar way the governing system for Model $\mathrm{V}$ can be constructed . Indeed, for the Model V, taking into account (14), (15), (5) from (6), (9), (8) we obtain correspondingly

$$
X_{3 \beta 0, \beta}+Q_{(+)} \sqrt{(\stackrel{(+)}{h}, 1)^{2}+(\stackrel{(+)}{h}, 2)^{2}+1}+\frac{1}{2 h}\left(X_{3 \beta 0} \stackrel{(-)}{h}, \beta-X_{330}\right)+\Phi_{30}=\rho \frac{\partial^{2} u_{30}}{\partial t^{2}},
$$




$$
\begin{aligned}
& X_{\alpha \beta 0, \beta}-\frac{1}{2 h}\left(X_{\alpha \beta 0} \stackrel{(+)}{h}, \beta-X_{\alpha 30}\right) \\
& +Q_{v_{\alpha}} \sqrt{(\stackrel{(-)}{h}, 1)^{2}+(\stackrel{(-)}{h}, 2)^{2}+1}+\Phi_{\alpha 0}=\rho \frac{\partial^{2} u_{\alpha 0}}{\partial t^{2}}, \quad \alpha=1,2 ; \\
& e_{330}=u_{3}\left(x_{1}, x_{2}, \stackrel{(+)}{h}, t\right)-u_{3}\left(x_{1}, x_{2}, \stackrel{(-)}{h}, t\right) \\
& =\frac{u_{30}}{2 h}-u_{3}\left(x_{1}, x_{2}, \stackrel{(-)}{h}, t\right)=\frac{v_{30}}{2}-u_{3}\left(x_{1}, x_{2}, \stackrel{(-)}{h}, t\right) \\
& e_{3 \beta 0}=\frac{1}{2}\left[u_{30, \beta}+u_{\beta}\left(x_{1}, x_{2}, \stackrel{(+)}{h}, t\right)-u_{\beta}\left(x_{1}, x_{2}, \stackrel{(-)}{h}, t\right)\right. \\
& \left.+u_{3}\left(x_{1}, x_{2}, \stackrel{(-)}{h}, t\right) \stackrel{(-)}{h}, \beta, u_{3}\left(x_{1}, x_{2}, \stackrel{(+)}{h}, t\right) \stackrel{(+)}{h}, \beta\right] \\
& =\frac{1}{2}\left[u_{30, \beta}+u_{\beta}\left(x_{1}, x_{2}, \stackrel{(+)}{h}, t\right)-\frac{1}{2 h} u_{\beta 0}\right. \\
& \left.+u_{3}\left(x_{1}, x_{2}, \stackrel{(-)}{h}, t\right) \stackrel{(-)}{h},{ }_{, \beta}-\frac{(+)}{h, \beta} u_{30}\right] \\
& =\frac{1}{2}\left[\left(h v_{30}\right), \beta-\frac{1}{2}\left(v_{\beta 0}+\stackrel{(+)}{h}, \beta, v_{30}\right)\right]+\chi_{3 \beta}, \quad \beta=1,2 . \\
& \chi_{3 \beta}:=\frac{1}{2}\left[u_{\beta}\left(x_{1}, x_{2}, \stackrel{(+)}{h}, t\right)+u_{3}\left(x_{1}, x_{2}, \stackrel{(-)}{h}, t\right) \stackrel{(-)}{h}, \underset{, \beta}{ }\right], \\
& e_{\alpha \beta 0}=\frac{1}{2}\left[u_{\alpha 0, \beta}+u_{\beta 0, \alpha}-u_{\beta}\left(x_{1}, x_{2}, \stackrel{(+)}{h}, t\right) \stackrel{(+)}{h},{ }_{\alpha}\right. \\
& \left.+u_{\beta}\left(x_{1}, x_{2}, \stackrel{(-)}{h}, t\right) \stackrel{(-)}{h},{ }_{\alpha}+u_{\alpha}\left(x_{1}, x_{2}, \stackrel{(-)}{h}, t\right) \stackrel{(-)}{h},{ }_{\beta}-u_{\alpha}\left(x_{1}, x_{2}, \stackrel{(+)}{h}, t\right) \stackrel{(+)}{h}, \beta\right] \\
& =\frac{1}{2}\left[u_{\alpha 0, \beta}+u_{\beta 0, \alpha}-u_{\beta}\left(x_{1}, x_{2}, \stackrel{(+)}{h}, t\right) \stackrel{(+)}{h},_{\alpha}+\frac{\stackrel{(-)}{h},{ }_{\alpha}}{2 h} u_{\beta 0}\left(x_{1}, x_{2}, t\right)\right. \\
& \left.+\frac{\stackrel{(-)}{h}, \beta}{2 h} u_{\alpha 0}\left(x_{1}, x_{2}, t\right)-u_{\alpha}\left(x_{1}, x_{2}, \stackrel{(+)}{h}, t\right) \stackrel{(+)}{h}, \beta\right] \\
& =\frac{1}{2}\left[\left(h v_{\alpha 0}\right)_{, \beta}+\left(h v_{\beta 0}\right)_{\alpha}+\frac{(-)}{h},_{\alpha} v_{\beta 0}+\frac{\stackrel{(-)}{h}, \beta}{2} v_{\alpha 0}\right]-\chi_{\alpha \beta} \text {, } \\
& \chi_{\alpha \beta}:=\frac{1}{2}\left[u_{\beta}\left(x_{1}, x_{2}, \stackrel{(+)}{h}, t\right) \stackrel{(+)}{h},_{\alpha}+u_{\alpha}\left(x_{1}, x_{2}, \stackrel{(+)}{h}, t\right) \stackrel{(+)}{h}, \beta\right], \quad \alpha, \beta=1,2 ;
\end{aligned}
$$

Substituting (46)-(48) into (7), we get

$$
\begin{gathered}
X_{330}=\lambda\left[\left(h v_{\gamma 0}\right),_{\gamma}+\frac{1}{2} \stackrel{(-)}{h}, \gamma v_{\gamma 0}-\chi_{\gamma \gamma}\right]+(\lambda+2 \mu)\left[\frac{1}{2} v_{30}-u_{3}\left(x_{1}, x_{2}, \stackrel{(-)}{h}, t\right)\right] \\
=\lambda\left[\left(h v_{\gamma 0}\right)_{\gamma}+\frac{1}{2} \frac{(-)}{h}, \gamma v_{\gamma 0}\right]+\frac{\lambda+2 \mu}{2} v_{30} \\
-\lambda \chi_{\gamma \gamma}-(\lambda+2 \mu) u_{3}\left(x_{1}, x_{2}, \stackrel{(-)}{h}, t\right), \\
X_{3 \beta 0}=\mu\left[\left(h v_{30}\right)_{\beta}-\frac{1}{2}\left(_{\beta 0}+\stackrel{(+)}{h}, v_{30}\right)\right]+2 \mu \chi_{3 \beta}, \quad \beta=1,2 ;
\end{gathered}
$$




$$
\begin{aligned}
& X_{\alpha \beta 0}=\lambda\left[\left(h v_{\gamma 0}\right), \gamma+\frac{1}{2} \stackrel{(-)}{h}, \gamma v_{\gamma 0}-\chi_{\gamma \gamma}+\frac{\lambda}{2} v_{30}-\lambda u_{3}\left(x_{1}, x_{2}, \stackrel{(-)}{h}, t\right)\right] \delta_{\alpha \beta} \\
& +\mu\left[\left(h v_{\alpha 0}\right)_{, \beta}+\left(h v_{\beta 0}\right)_{\alpha}+\frac{(-)}{h},_{\alpha} v_{\beta 0}+\frac{\stackrel{(-)}{h}, \beta}{2} v_{\alpha 0}\right]-2 \mu \chi_{\alpha \beta} \\
& =\lambda\left[\left(h v_{\gamma 0}\right)_{\gamma}+\frac{\stackrel{(-)}{h},,_{\gamma}}{2} v_{\gamma 0}+\frac{v_{30}}{2}\right] \delta_{\alpha \beta}+\mu\left[\left(h v_{\alpha 0}\right)_{\beta}+\left(h v_{\beta 0}\right)_{, \alpha}+\frac{\stackrel{(-)}{h},,_{\alpha}}{2} v_{\beta 0}\right. \\
& \left.+\frac{\stackrel{(-)}{h}, \beta}{2} v_{\alpha 0}\right]-\lambda\left[\chi_{\gamma \gamma}+u_{3}\left(x_{1}, x_{2}, \stackrel{(-)}{h}, t\right)\right] \delta_{\alpha \beta}-2 \mu \chi_{\alpha \beta}, \quad \alpha, \beta=1,2 .
\end{aligned}
$$

Substituting (49)-(51) into (45), (44) we have the following governing system for Model V

$$
\begin{aligned}
& \mu\left(h v_{\alpha 0}\right)_{, \beta \beta}+\mu\left(h v_{\beta 0}\right)_{\alpha \beta}+\lambda\left(h v_{\gamma 0}\right)_{, \alpha}+\frac{\lambda}{2}\left(\stackrel{(-)}{h}, \gamma v_{\gamma 0}\right), \alpha+\frac{\mu}{2}\left(\stackrel{(-)}{h},_{\alpha} v_{\beta 0}\right), \beta \\
& +\frac{\mu}{2}\left(\stackrel{(-)}{h},{ }_{\beta} v_{\alpha 0}\right), \beta+\frac{\lambda}{2} v_{30, \alpha}-\frac{1}{2 h}\left\{\left[\lambda\left(\left(h v_{\gamma 0}\right)_{\gamma}+\frac{\stackrel{(-)}{h}, \gamma}{2} v_{\gamma 0}+\frac{v_{30}}{2}\right) \delta_{\alpha \beta}\right.\right.
\end{aligned}
$$

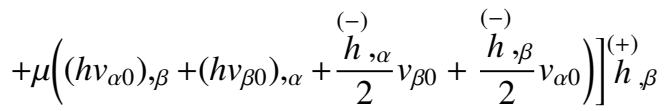

$$
\begin{aligned}
& \left.-\mu\left[\left(h v_{30}\right),_{\alpha}-\frac{1}{2}\left(v_{\alpha 0}+\stackrel{(+)}{h},{ }_{\alpha} v_{30}\right)\right]\right\}+Q_{\left(v_{\alpha}\right)} \sqrt{\left(\stackrel{(-)}{h},{ }_{1}\right)^{2}+(\stackrel{(-)}{h}, 2)^{2}+1} \\
& -\lambda\left[\chi_{\gamma \gamma}+u_{3}\left(x_{1}, x_{2}, \stackrel{(-)}{h}, t\right)\right]{ }_{\alpha}-2 \mu \chi_{\alpha \beta, \beta}+\frac{\lambda}{2 h}\left[\left(\chi_{\gamma \gamma}+u_{3}\left(x_{1}, x_{2}, \stackrel{(-)}{h}, t\right)\right) \delta_{\alpha \beta}\right. \\
& \left.-2 \mu \chi_{\alpha \beta}\right]_{h, \beta}^{(+)}+\frac{\mu}{h} \chi_{3 \alpha}+\Phi_{\alpha 0}=\rho h \frac{\partial^{2} v_{\alpha 0}}{\partial t^{2}}, \quad \alpha=1,2, \\
& \left.\mu\left(h v_{30}\right)_{, \beta \beta}-\frac{\mu}{2} v_{\beta 0, \beta}-\frac{\mu}{2}\left(\stackrel{(+)}{h},{ }_{, \beta} v_{30}\right)\right)_{\beta}+\frac{1}{2 h}\left\{\mu\left[\left(h v_{30}\right)_{, \beta}-\frac{1}{2}\left(v_{\beta 0}+\stackrel{(+)}{h},{ }_{, \beta} v_{30}\right)\right] \stackrel{(-)}{h}, \beta\right.
\end{aligned}
$$

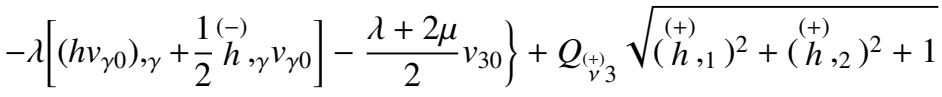

$$
\begin{aligned}
& +2 \mu \chi_{3 \beta, \beta}+\frac{\mu}{h} \chi_{3 \beta} \stackrel{(-)}{h}, \beta+\frac{1}{2 h}\left[\lambda \chi_{\gamma \gamma}+(\lambda+2 \mu) u_{3}\left(x_{1}, x_{2}, \stackrel{(-)}{h}, t\right)\right]+\Phi_{30}=\rho h \frac{\partial^{2} v_{30}}{\partial t^{2}} .
\end{aligned}
$$

Taking into account conditions of Model VII and (14), (15), from (6), (9), (8) we obtain correspondingly

$$
\begin{aligned}
& X_{i \beta 0, \beta}+Q_{\stackrel{(+)}{i}_{i}} \sqrt{(\stackrel{(+)}{h}, 1,)^{2}+(\stackrel{(+)}{h}, 2)^{2}+1} \\
& +\frac{1}{2 h}\left(X_{i \beta 0} \stackrel{(-)}{h}, \beta_{,}-X_{i 30}\right)+\Phi_{i 0}=\rho \frac{\partial^{2} u_{i 0}}{\partial t^{2}}, \quad i=1,2,3 \text {; } \\
& e_{330}=\frac{u_{30}}{2 h}-u_{3}\left(x_{1}, x_{2}, \stackrel{(-)}{h}, t\right)=\frac{v_{30}}{2}-u_{3}\left(x_{1}, x_{2}, \stackrel{(-)}{h}, t\right), \\
& e_{3 \beta 0}=\frac{1}{2}\left[u_{30, \beta}+\frac{u_{\beta 0}}{2 h}-u_{\beta}\left(x_{1}, x_{2}, \stackrel{(-)}{h}, t\right)+u_{3}\left(x_{1}, x_{2}, \stackrel{(-)}{h}, t\right) \stackrel{(-)}{h}, \beta\right. \\
& \left.-\frac{u_{30}}{2 h} \stackrel{(+)}{h}, \beta\right]=\frac{1}{2}\left[\left(h v_{30}\right)_{, \beta}+\frac{v_{\beta 0}}{2}-\frac{v_{30}}{2} \stackrel{(+)}{h}, \beta\right]-\frac{1}{2}\left[u_{\beta}\left(x_{1}, x_{2}, \stackrel{(-)}{h}, t\right)\right. \\
& \left.-u_{3}\left(x_{1}, x_{2}, \stackrel{(-)}{h}, t\right) \stackrel{(-)}{h}, \beta\right], \quad \beta=1,2,
\end{aligned}
$$




$$
\begin{aligned}
& e_{\alpha \beta 0}=\frac{1}{2}\left[u_{\alpha 0, \beta}+u_{\beta 0, \alpha}-\stackrel{(+)}{h},{ }_{\alpha} \frac{u_{\beta 0}}{2 h}+u_{\beta}\left(x_{1}, x_{2}, \stackrel{(-)}{h}, t\right) \stackrel{(-)}{h}, \alpha\right. \\
& \left.+u_{\alpha}\left(x_{1}, x_{2}, \stackrel{(-)}{h}, t\right) \stackrel{(-)}{h},,_{\beta}-\stackrel{(+)}{h}, \frac{u_{\alpha 0}}{2 h}\right] \\
& =\frac{1}{2}\left[\left(h v_{\alpha 0}\right)_{, \beta}+\left(h v_{\beta 0}\right)_{\alpha}-\frac{\stackrel{(+)}{h},_{\alpha}}{2} v_{\beta 0}-\frac{\stackrel{(+)}{h}, \beta}{2} v_{\alpha 0}\right] \\
& +\frac{1}{2}\left[u_{\beta}\left(x_{1}, x_{2}, \stackrel{(-)}{h}, t\right) \stackrel{(-)}{h}, \alpha+u_{\alpha}\left(x_{1}, x_{2}, \stackrel{(-)}{h}, t\right) \stackrel{(-)}{h}, \beta\right], \quad \alpha, \beta=1,2 .
\end{aligned}
$$

Substituting (55)-(57) into (7), we get

$$
\begin{aligned}
& X_{330}=\lambda\left[\left(h v_{\gamma 0}\right),{ }_{\gamma}-\frac{1}{2} \stackrel{(+)}{h},{ }_{\gamma} v_{\gamma 0}+\frac{v_{30}}{2}\right]+\lambda\left[u_{\gamma}\left(x_{1}, x_{2}, \stackrel{(-)}{h}, t\right) \stackrel{(-)}{h}, \gamma\right. \\
& \left.-u_{3}\left(x_{1}, x_{2}, \stackrel{(-)}{h}, t\right)\right]+\mu v_{30}-2 \mu u_{3}\left(x_{1}, x_{2}, \stackrel{(-)}{h}, t\right), \\
& X_{3 \beta 0}=\mu\left[\left(h v_{30}\right)_{, \beta}+\frac{1}{2} v_{\beta 0}-\frac{(+)}{h, \beta} v_{30}\right] \\
& -\mu\left[u_{\beta}\left(x_{1}, x_{2}, \stackrel{(-)}{h}, t\right)-u_{3}\left(x_{1}, x_{2}, \stackrel{(-)}{h}, t\right) \stackrel{(-)}{h}, \beta\right] \quad \beta=1,2, \\
& X_{\alpha \beta 0}=\lambda\left[\left(h v_{\gamma 0}\right)_{\gamma}-\frac{1}{2} \stackrel{(+)}{h}, \gamma v_{\gamma 0}+\frac{v_{30}}{2}\right] \delta_{\alpha \beta}+\lambda\left[u_{\gamma}\left(x_{1}, x_{2}, \stackrel{(-)}{h}, t\right) \stackrel{(-)}{h}, \gamma\right. \\
& \left.-u_{3}\left(x_{1}, x_{2}, \stackrel{(-)}{h}, t\right)\right] \delta_{\alpha \beta}+\mu\left[\left(h v_{\alpha 0}\right)_{, \beta}+\left(h v_{\beta 0}\right)_{, \alpha}-\stackrel{\stackrel{(+)}{h},{ }_{\alpha}}{2} v_{\beta 0}-\frac{\stackrel{(+)}{h}, \beta}{2} v_{\alpha 0}\right] \\
& +\mu\left[u_{\beta}\left(x_{1}, x_{2}, \stackrel{(-)}{h}, t\right) \stackrel{(-)}{h},_{\alpha}+u_{\alpha}\left(x_{1}, x_{2}, \stackrel{(-)}{h}, t\right) \stackrel{(-)}{h}, \beta\right], \quad \alpha, \beta=1,2 .
\end{aligned}
$$

Substituting (58), (60) into (54), we have the following system for Model VII

$$
\begin{aligned}
& \mu\left(h v_{\alpha 0}\right)_{\beta \beta}+\mu\left(h v_{\beta 0}\right),_{\alpha \beta}+\lambda\left(h v_{\gamma 0}\right)_{, \alpha}-\frac{\lambda}{2}\left(\stackrel{+}{h}, \gamma, v_{\gamma 0}\right)_{, \alpha}+\frac{\lambda}{2} v_{30, \alpha}-\frac{\mu}{2}\left(\stackrel{(+)}{h},,_{\alpha} v_{\beta 0}\right)_{, \beta} \\
& -\frac{\mu}{2}\left(\stackrel{+}{h},{ }_{\beta} v_{\alpha 0}\right)_{, \beta}+\frac{1}{2 h}\left\{\left[\lambda\left(\left(h v_{\gamma 0}\right)_{\gamma}-\frac{1}{2} \stackrel{(+)}{h}, \gamma v_{\gamma 0}+\frac{v_{30}}{2}\right) \delta_{\alpha \beta}\right.\right.
\end{aligned}
$$

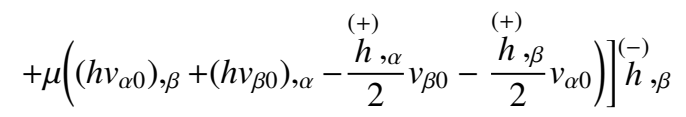

$$
\begin{aligned}
& \left.-\mu\left(\left(h v_{30}\right),_{\alpha}+\frac{1}{2} v_{\alpha 0}-\frac{\stackrel{(+)}{h}, \alpha}{2} v_{30}\right)\right\}+Q_{(+)} \sqrt{\left(\stackrel{(+)}{h,,_{1}}\right)^{2}+(\stackrel{+)}{h}, 2)^{2}+1} \\
& +\lambda\left[u_{\gamma}\left(x_{1}, x_{2}, \stackrel{(-)}{h}, t\right) \stackrel{(-)}{h}, \gamma-u_{3}\left(x_{1}, x_{2}, \stackrel{(-)}{h}, t\right)\right], \alpha \\
& +\mu\left[u_{\beta}\left(x_{1}, x_{2}, \stackrel{(-)}{h}, t\right) \stackrel{(-)}{h}, \alpha+u_{\alpha}\left(x_{1}, x_{2}, \stackrel{(-)}{h}, t\right) \stackrel{(-)}{h}, \beta\right], \beta \\
& +\frac{1}{2 h}\left[\lambda\left(u_{\gamma}\left(x_{1}, x_{2}, \stackrel{(-)}{h}, t\right) \stackrel{(-)}{h}, \gamma-u_{3}\left(x_{1}, x_{2}, \stackrel{(-)}{h}, t\right)\right) \delta_{\alpha \beta}\right. \\
& \left.+\mu\left(u_{\beta}\left(x_{1}, x_{2}, \stackrel{(-)}{h}, t\right) \stackrel{(-)}{h},{ }_{\alpha}+u_{\alpha}\left(x_{1}, x_{2}, \stackrel{(-)}{h}, t\right) \stackrel{(-)}{h}, \beta\right)\right]^{(-)} h, \beta \\
& +\frac{\mu}{2 h}\left(u_{\alpha}\left(x_{1}, x_{2}, \stackrel{(-)}{h}, t\right)-u_{3}\left(x_{1}, x_{2}, \stackrel{(-)}{h}, t\right) \stackrel{(-)}{h}, \alpha\right)+\Phi_{\alpha 0}=\rho h \frac{\partial^{2} v_{\alpha 0}}{\partial t^{2}}, \quad \alpha=1,2,
\end{aligned}
$$




$$
\begin{aligned}
& \mu\left(h v_{30}\right)_{, \beta \beta}+\frac{\mu}{2} v_{\beta 0, \beta}-\frac{\mu}{2}\left(\stackrel{(+)}{h}, \beta, v_{30}\right), \beta+\frac{1}{2 h}\left\{\mu\left[\left(h v_{30}\right)_{, \beta}+\frac{1}{2} v_{\beta 0}-\frac{\stackrel{(+)}{h}, \frac{\beta}{2}}{2} v_{30}\right]^{(-)} h,\right. \\
& \left.-\lambda\left[\left(h v_{\gamma 0}\right)_{\gamma}-\frac{1}{2} \stackrel{(+)}{h}, v_{\gamma} v_{\gamma 0}\right]-\frac{\lambda+2 \mu}{2} v_{30}\right\}+Q_{\stackrel{(+)}{3}} \sqrt{\left(\stackrel{(+)}{h},,_{1}\right)^{2}+(\stackrel{(+)}{h}, 2)^{2}+1} \\
& -\mu\left[u_{\beta}\left(x_{1}, x_{2}, \stackrel{(-)}{h}, t\right)-u_{3}\left(x_{1}, x_{2}, \stackrel{(-)}{h}, t\right) \stackrel{(-)}{h}, \beta\right], \beta \\
& -\frac{1}{2 h}\left\{\mu\left[u_{\beta}\left(x_{1}, x_{2}, \stackrel{(-)}{h}, t\right)-u_{3}\left(x_{1}, x_{2}, \stackrel{(-)}{h}, t\right) \stackrel{(-)}{h},{ }_{, \beta}^{(-)}\right]_{, \beta}\right. \\
& \left.-\lambda\left[u_{\gamma}\left(x_{1}, x_{2}, \stackrel{(-)}{h}, t\right) \stackrel{(-)}{h}, \gamma-u_{3}\left(x_{1}, x_{2}, \stackrel{(-)}{h}, t\right)\right]+2 \mu u_{3}\left(x_{1}, x_{2}, \stackrel{(-)}{h}, t\right)\right\}+\Phi_{30}=\rho h \frac{\partial^{2} v_{30}}{\partial t^{2}} .
\end{aligned}
$$

Model VI and Model VIII there follow from the Model V and Model VII after changing direction of the $x_{3}-$ axis. The well-known governing systems of the Model I and Model II have the forms [1-3]

$$
\begin{aligned}
& \mu\left[\left(h v_{\alpha 0, \beta}\right)_{, \beta}+\left(h v_{\beta 0, \alpha}\right)_{, \beta}\right]+\lambda\left(h v_{\gamma 0, \gamma}\right)_{, \alpha}+Q_{\dot{v}_{\alpha}} \sqrt{(\stackrel{(+)}{h}, 1)^{2}+(\stackrel{(+)}{h, 2})^{2}+1} \\
& +Q_{(-)} \sqrt{(\stackrel{(-)}{h}, 1)^{2}+(\stackrel{(-)}{h}, 2)^{2}+1}+\Phi_{\alpha 0}=\rho h \frac{\partial^{2} v_{\alpha 0}}{\partial t^{2}}, \quad \alpha=1,2, \\
& \mu\left(h v_{30, \beta}\right)_{, \beta}+Q_{(+)} \sqrt{(\stackrel{(+)}{h}, 1)^{2}+(\stackrel{+)}{h}, 2)^{2}+1} \\
& +Q_{(-)} \sqrt{(\stackrel{(-)}{h}, 1)^{2}+(\stackrel{(-)}{h}, 2)^{2}+1}+\Phi_{30}=\rho h \frac{\partial^{2} v_{30}}{\partial t^{2}}
\end{aligned}
$$

and [3]

$$
\begin{aligned}
& \mu\left(h v_{\alpha 0}\right)_{, \beta \beta}+(\lambda+\mu)\left(h v_{\gamma 0}\right)_{, \alpha}-(\ln h)_{, \beta}\left\{\lambda \delta_{\alpha \beta}\left(h v_{\gamma 0}\right)_{, \gamma}\right. \\
& \left.+\mu\left[\left(h v_{\alpha 0}\right)_{, \beta}+\left(h v_{\beta 0}\right)_{, \alpha}\right]\right\}+2 \mu \Psi_{\alpha \beta, \beta}\left(x_{1}, x_{2}, t\right)+\lambda \Psi_{k k, \alpha}\left(x_{1}, x_{2}, t\right)-(\ln h)_{, \beta} \\
& \times\left[\lambda \delta_{\alpha \beta} \Psi_{k k}\left(x_{1}, x_{2}, t\right)+2 \mu \Psi_{\alpha \beta}\left(x_{1}, x_{2}, t\right)\right]+\Phi_{\alpha 0}\left(x_{1}, x_{2}, t\right)=\rho h \frac{\partial^{2} v_{\alpha 0}}{\partial t^{2}}, \quad \alpha=1,2 \\
& \mu\left(h v_{30}\right)_{, \beta \beta}-(\ln h)_{, \beta} \mu\left(h v_{30}\right)_{, \beta}+2 \mu \Psi_{3 \beta, \beta}\left(x_{1}, x_{2}, t\right) \\
& -2 \mu(\ln h)_{, \beta} \Psi_{3 \beta}\left(x_{1}, x_{2}, t\right)+\Phi_{30}\left(x_{1}, x_{2}, t\right)=\rho h \frac{\partial^{2} v_{30}}{\partial t^{2}}
\end{aligned}
$$

where

$$
\begin{gathered}
\Psi_{3 \beta}\left(x_{1}, x_{2}, t\right):= \\
\left.\frac{1}{2}\left[u_{3}\left(x_{1}, x_{2}, \stackrel{(-)}{h}, t\right) \stackrel{(-)}{h}{ }_{, \beta}-u_{3}\left(x_{1}, x_{2}, \stackrel{(+)}{h}, t\right) \stackrel{(+)}{h}\right)_{, \beta}+u_{\beta}\left(x_{1}, x_{2}, \stackrel{(+)}{h}, t\right)-u_{\beta}\left(x_{1}, x_{2}, \stackrel{(-)}{h}, t\right)\right],
\end{gathered}
$$

respectively.

Similarly, we construct the following models:

Model IX.

On the upper surface $\stackrel{(+)}{h}$ stress vector is known, on the lower surface $\stackrel{(-)}{h}$ the quantities (10) are known.

Model X.

On the upper surface $\stackrel{(+)}{h}$ stress vector is known, on the lower surface $\stackrel{(-)}{h}$ the quantities (11) are known.

Model XI.

$$
\text { On the upper surface } \stackrel{(+)}{h} \text { displacements are known, }
$$


on the lower surface $\stackrel{(-)}{h}$ the quantities (10) are known.

Model XII.

On the upper surface $\stackrel{(+)}{h}$ displacements are known,

on the lower surface $\stackrel{(-)}{h}$ the quantities (11) are known.

Model XIII.

On the upper surface $\stackrel{(+)}{h}$ the quantities (10) are known, on the lower surface $\stackrel{(-)}{h}$ stress vector is known.

Model XIV.

On the upper surface $\stackrel{(+)}{h}$ the quantities (11) are known, on the lower surface $\stackrel{(-)}{h}$ stress vector is known.

Model XV.

On the upper surface $\stackrel{(+)}{h}$ the quantities (10) are known, on the lower surface $\stackrel{(-)}{h}$ displacements are known.

Model XVI.

On the upper surface $\stackrel{(+)}{h}$ the quantities (11) are known, on the lower surface $\stackrel{(-)}{h}$ displacements are known.

Similar hierarchical models can be constructed for composite prismatic shells as well (see Section 4 below).

\section{Analysis of the Constructed Models}

Models III-VIII are actually suggested in the present paper for the first time and, therefore, are not studied at all. Model II, actually, is not investigated. Model I is studied sufficiently well even in the case of cusped prismatic shells, i.e., in the case of prismatic shells with a cusped edge $\omega_{0} \subseteq \partial \omega$, where the thickness $2 h\left(x_{1}, x_{2}\right)$ vanishes:

$$
\omega_{0}:=\left\{\left(x_{1}, x_{2}\right) \in \partial \omega: 2 h\left(x_{1}, x_{2}\right)=0\right\}
$$

Evidently, $\omega_{0}$ is a closed set.

Dirichlet Problem. Find a solution $v_{i 0} \in C^{2}(\omega) \cap C(\bar{\omega}), i=1,2,3$, of the governing system in $\omega$, satisfying the boundary conditions

$$
v_{i 0}\left(x_{1}, x_{2}\right)=\varphi_{i}\left(x_{1}, x_{2}\right), \quad\left(x_{1}, x_{2}\right) \in \partial \omega, \quad i=1,2,3,
$$

where $\varphi_{i}, i=1,2,3$, are given continuous on $\partial \omega$ functions.

Keldysh Problem. Find a bounded solution $v_{i 0} \in C^{2}(\omega) \cap C\left(\omega \backslash \omega_{0}\right), i=1,2,3$, of the governing system in $\omega$, satisfying the boundary conditions

$$
v_{i 0}\left(x_{1}, x_{2}\right)=\varphi_{i}\left(x_{1}, x_{2}\right), \quad\left(x_{1}, x_{2}\right) \in \partial \omega \backslash \omega_{0}, \quad i=1,2,3,
$$

where $\varphi_{i}, i=1,2,3$, are given continuous on $(\partial \omega) \backslash \omega_{0}$ functions.

Here $C^{2}$ is a class of twice continuously differentiable functions in the domain under consideration; $C$ is a class of continuous functions on the sets under consideration.

Considering cusped prismatic shells within the framework of models I-XVI, governing systems are systems of partial differential equations with order degeneration. Since the governing systems are not degenerate with respect to $t$ by $t=0$, setting initial conditions do not posses peculiarities in contrast to setting boundary conditions with respect to space variables at cusped edges. 
The question of consideration of prismatic shells with cusped edges within the framework of Model I was raised by I. Vekua [1, 2]. Concerning studies of the governing system of the general $N$-th $\left(N=N_{1}=N_{2}=N_{3}\right)$ order approximation see [3] and references therein. System (63), (64) was explored in [16]-[18] (see also [3] and references therein), where the main peculiarities of the well-posedeness of boundary conditions in displacements (i.e. for $v_{i 0}$, $i=1,2,3)$ are established:

on the non-cusped edge $\partial \omega \backslash \omega_{0}$ boundary conditions can always be prescribed;

on the cusped edge $\omega_{0}$

(i) boundary conditions should be prescribed (Dirichlet type problem) if

$$
\frac{\partial h}{\partial v}=+\infty
$$

(ii) boundary conditions should not be prescribed (Keldysh type problem) if

$$
\frac{\partial h}{\partial v} \geq 0
$$

where $v$ is the inward normal to $\omega_{0} \subseteq \partial \omega$.

Criterion in the integral form is given in [18]:

on the cusped edge $\omega_{0}$

(i) boundary conditions should be prescribed if

$$
\int_{P}^{Q} h^{-1} d v<+\infty
$$

(ii) boundary conditions should not be prescribed if

$$
\int_{P}^{Q} h^{-1} d v=+\infty,
$$

where $P \in \omega_{0}, Q \in \omega$.

The last criterion in the $N$-th approximation looks like:

on the cusped edge boundary conditions for

$$
v_{i r}, \quad i=1,2,3, \quad r=0,1, \ldots, N,
$$

should be prescribed if

$$
\int_{P}^{Q} h^{-2 r-1} d v<+\infty
$$

and should not be prescribed if

$$
\int_{P}^{Q} h^{-2 r-1} d v=+\infty .
$$

Let us note that as it is easily seen from systems (63), (64); (65), (66); (24), (25), and (42), (43), systems (63), (65), (24), and (42) with respect to $v_{\alpha 0}, \quad \alpha=1,2$, and equations (64), (66), (25), and (43) with respect to $v_{30}$ we can consider separately.

If the thickness has the form

$$
2 h\left(x_{1}, x_{2}\right)=h_{0} x_{2}^{\kappa}, \quad h_{0}=\text { const }>0, \quad \kappa=\text { const } \geq 0,
$$


and $\partial \omega$ contains a segment $\omega_{0}$ of the $x_{1}$-axis, then (67), (69) mean that $0 \leq \kappa<1$, while (68), (70) mean that $\kappa \geq 1$. Evidently, if $0 \leq \kappa<1$, a profile (a normal cross-section of the prismatic shell at the cusped edge) has a smooth boundary, while if $\kappa \geq 1$, the profile is not smooth, namely, ends with an angle $\varphi \in[0, \pi$ [ at cusped edge.

Cusped prismatic shells of the form (71) are investigated at most (see [3], [14], [15], [19] and the references given there). When $\omega$ is a half-plane $x_{2} \geq 0$, the Flamant, Cerutti, and Carothers type problems are solved in explicit forms, which in the particular case $\kappa=0$ coincide with the classical Flamant, Cerutti, and Carothers formulas for the plate of a constant thickness [20]-[23]. These investigations are carried out within the framework of Model I (i.e., for system (63), (64)).

Elastic equilibrium of a cusped prismatic shell under action of an arbitrary stress vector concentrated along the cusped edge is solved in the explicit form (quadratures) [24].

System (42) (Model IV) provided $\Psi_{33} \equiv 0$ and system (63) (Model I) coincide.

System (65) (Model II) provided $\Psi_{33} \equiv 0$ and system (24) (Model III) coincide.

So, systems (42) and (63) coincide up to the known summand $\Psi_{33}$. Therefore, all the results obtained for system (63) can appropriately be reformulated for system (42).

Note that systems (42) and (63) for the symmetric $\left.\stackrel{(-)}{h}\left(x_{1}, x_{2}\right)=-\stackrel{(+)}{h}\left(x_{1}, x_{2}\right)\right)$ prismatic shells of a constant thickness $2 h=$ const coincide with the system of the plane strain.

Systems (24) and (65) coincide up to the known summand $\Psi_{33, \alpha}, \quad \alpha=1,2$. Therefore, systems (24) and (65) can be studied by the same methods and the results will be qualitatively the same (e.g. in the sense of well-posedeness of boundary value problems).

Equation (25) (Model III) and equation (64) (Model I) coincide up to the known summand

$$
\mu\left[u_{\beta}\left(x_{1}, x_{2}, \stackrel{(+)}{h}, t\right)-u_{\beta}\left(x_{1}, x_{2}, \stackrel{(-)}{h}, t\right)\right]_{, \beta} .
$$

Hence, all the results obtained for equation (64) can appropriately be reformulated for equation (25).

Equations (43) (Model IV) and (66) (Model II) coincide up to their known parts. Therefore, they can be studied by the same methods and the results will be qualitatively the same (e.g. in the sense of well-posedeness of boundary value problems).

Similar analysis can be carried out with respect to Models V-XVI.

Considering models I-XVI in the case (71) with $\partial \omega$ containing a segment of the $x_{1}$-axis, we can take arbitrarily functions $\stackrel{(+)}{h}\left(x_{1}, x_{2}\right)$ and $\stackrel{(-)}{h}\left(x_{1}, x_{2}\right)$, provided their difference $\stackrel{(+)}{h}\left(x_{1}, x_{2}\right)-\stackrel{(-)}{h}\left(x_{1}, x_{2}\right)$, i.e., thickness varies according to (71). In particular, (71) will be realized if we assume

$$
\begin{aligned}
\stackrel{(+)}{h}\left(x_{1}, x_{2}\right)= & \stackrel{(+)}{h} x_{2}^{\kappa}, \quad \stackrel{(-)}{h}\left(x_{1}, x_{2}\right)=\stackrel{(-)}{h} x_{2}^{\kappa}, \quad \kappa=\text { const } \geq 0, \\
& \stackrel{(+)}{h_{0}}, \stackrel{(-)}{h}=\text { const }, \quad \stackrel{(+)}{h} \stackrel{(-)}{h}_{0}-0 .
\end{aligned}
$$

If $\kappa>0$, we have to do with a cusped prismatic shell with a cusped edge by $x_{2}=0$. If $\kappa=0$, the prismatic shell will be of a constant thickness $h_{0}$, which in the symmetric case $\stackrel{(-)}{h}=-\stackrel{(+)}{h}$ will be a plate.

The following theorem is true [25] (compare with [26], where $m_{1}=0$ )

Theorem 2. If the coefficients $a_{\alpha}, \alpha=1,2$, and $c$ of the equation

$$
x_{2}^{m_{\alpha}} u,_{\alpha \alpha}+a_{\alpha}\left(x_{1}, x_{2}\right) u,_{\alpha}+c\left(x_{1}, x_{2}\right) u=0, \quad c \leq 0, \quad m_{\alpha}=\text { const } \geq 0, \quad \alpha=1,2,
$$

are analytic in $\bar{\omega}$ bounded by a sufficiently smooth arc $\left(\partial \omega \backslash \omega_{0}\right)$ lying in the half-plane $x_{2} \geq 0$ and by a segment $\omega_{0}$ of the $x_{1}$-axis, then

(i) if either $m_{2}<1$, or $m_{2} \geq 1, a_{2}\left(x_{1}, x_{2}\right)<x_{2}^{m_{2}-1}$ in $\bar{I}_{\delta}$ for some $\delta=$ const $>0$, where

$$
I_{\delta}:=\left\{\left(x_{1}, x_{2}\right) \in \omega: 0<x_{2}<\delta\right\},
$$

the Dirichlet problem is correct;

(ii) if $m_{2} \geq 1, a_{2}\left(x_{1}, x_{2}\right) \geq x_{2}^{m_{2}-1}$ in $I_{\delta}$ and $a_{1}\left(x_{1}, x_{2}\right)=O\left(x_{2}^{m_{1}}\right), x_{2} \rightarrow 0_{+}(O$ is the Landau symbol $)$, the Keldysh problem is correct. 
Remark 3. If $1<m_{2}<2, b(x, 0) \leq 0$, the Dirichlet problem is correct.

Using the method applied in [27] (see pages 58, 68-74), it is not difficult to verify that the theorem is also true for Hölder continuous $c$ and $a_{\alpha}, \alpha=1,2$, on $\bar{\omega}$, provided:

(i) $\lim _{x_{2} \rightarrow 0_{+}} x_{2}^{1-m_{2}} a_{2}\left(x_{1}, x_{2}\right)=a_{0}=$ const $<1$ for $\left(x_{1}, 0\right) \in \omega_{0}$ when $0 \leq m_{2}<1$;

(ii) if $a_{2}\left(x_{1}^{0}, 0\right)=0$ for a fixed $\left(x_{1}^{0}, 0\right) \in \omega_{0}$ when $1<n<2$, then there exists such a $\delta=$ const $>0$ that $a_{2}\left(x_{1}^{0}, x_{2}\right)=\kappa\left(x_{1}^{0}, x_{2}\right) \cdot x_{2}$ with bounded $\kappa\left(x_{1}^{0}, x_{2}\right)$ for $0 \leq x_{2}<\delta$.

Since in each of Models I and II qualitative properties by setting boundary conditions for $v_{10}, v_{20}$, and $v_{30}$ are the same, in order to compare Model I and Model II for the sake of clearness and simplicity we assume $v_{10} \equiv 0,\left(u_{1} \equiv 0\right)$, $v_{20} \equiv 0,\left(u_{2} \equiv 0\right), v_{30}=v_{30}\left(x_{2}\right)$, then we get

$$
\left.\left(x_{2}^{K} v_{30,2}\right)_{, 2}+T_{1}\left(x_{2}\right)=0, \quad x_{2} \in\right] 0, L[, \quad L=\text { const }>0,
$$

for Model I (by the way also for Model III),

$$
\left.\left(x_{2}^{\kappa} v_{30}\right)_{, 22}-\kappa x_{2}^{-1}\left(x_{2}^{\kappa} v_{30}\right)_{, 2}+T_{2}\left(x_{2}\right)=0, \quad x_{2} \in\right] 0, L[, \quad L=\text { const }>0,
$$

for Model II (by the way also for Model IV), where

$$
\begin{gathered}
\mu \frac{h_{0}}{2} T_{1}\left(x_{2}\right):=Q_{v_{\nu}} \sqrt{(\stackrel{(+)}{h}, 2)^{2}+1}+Q_{(-)} \sqrt{(\stackrel{(-)}{h}, 2)^{2}+1}+\Phi_{30}, \\
\mu \frac{h_{0}}{2} T_{2}\left(x_{2}\right):=\mu\left[u_{3}\left(x_{2}, \stackrel{(-)}{h}, t\right) \stackrel{(-)}{h}, 2-u_{3}\left(x_{2}, \stackrel{(+)}{h}, t\right) \stackrel{(+)}{h}, 2\right], 2 \\
-\mu \kappa x_{2}^{-1}\left[u_{3}\left(x_{2}, \stackrel{(-)}{h}, t\right) \stackrel{(-)}{h}, 2-u_{3}\left(x_{2}, \stackrel{(+)}{h}, t\right) \stackrel{(+)}{h}, 2\right]+\Phi_{30}
\end{gathered}
$$

are known.

Note that for Model V and Model VII we have

$$
\left(x_{2}^{\kappa} v_{30}\right)_{, 22}-\frac{1}{h_{0}}\left(\stackrel{(+)}{h}, 2 v_{30}\right)_{, 2}+\frac{x_{2}^{-\kappa}}{\mu h_{0}^{2}}\left\{\mu\left[h_{0}\left(x_{2}^{\kappa} v_{30}\right)_{, 2}-\stackrel{(+)}{h}, 2 v_{30}\right] \stackrel{(-)}{h}, \frac{\lambda+2 \mu}{2} v_{30}\right\}+T_{3}\left(x_{2}\right)=0
$$

where

$$
\begin{aligned}
& \mu \frac{h_{0}}{2} T_{3}\left(x_{2}\right):=\mu\left[u_{3}\left(x_{2}, \stackrel{(-)}{h}, t\right) \stackrel{(-)}{h, 2}\right], 2+Q_{v_{3}} \sqrt{(\stackrel{(+)}{h}, 2)^{2}+1} \\
& +\frac{x_{2}^{-\kappa}}{h_{0}}\left[\mu u_{3}\left(x_{2}, \stackrel{(-)}{h}, t\right)(\stackrel{(-)}{h}, 2)^{2}+(\lambda+2 \mu) u_{3}\left(x_{2}, \stackrel{(-)}{h}, t\right)\right]+\Phi_{30}
\end{aligned}
$$

is known. In particular,

$$
\begin{gathered}
x_{2}^{2} v_{30,22}+\kappa x_{2} v_{30,2}+\left\{\kappa\left[\frac{\stackrel{(+)}{h_{0}}}{h_{0}}\left(1-\kappa \frac{(-)}{h_{0}} h_{0}\right)-1\right]\right. \\
\left.-\frac{\lambda+2 \mu}{\mu h_{0}^{2}} x_{2}^{2(1-\kappa)}\right\} v_{30}-x_{2}^{2-\kappa} T_{3}\left(x_{2}\right)=0, \\
\mu \frac{h_{0}}{2} T_{3}\left(x_{2}\right):=\mu\left[u_{3}\left(x_{2}, \stackrel{(-)}{h}, t\right) \kappa h_{0} \chi_{2}^{\kappa-1}\right], 2+Q_{\nu 3} \sqrt{\left(\kappa h_{0}\right)^{2} \chi_{2}^{2(\kappa-1)}+1} \\
+\frac{(-)}{\mu\left(\kappa h_{0}\right)^{2} x_{2}^{\kappa-2}} \\
h_{0} u_{3}\left(x_{2}, \stackrel{(-)}{h}, t\right)+(\lambda+2 \mu) \frac{x_{2}^{-\kappa}}{h_{0}} u_{3}\left(x_{2}, \stackrel{(-)}{h}, t\right)+\Phi_{30} .
\end{gathered}
$$


General solutions of equations (72) and (73) have the forms

$$
\begin{gathered}
v_{30}^{I}\left(x_{2}\right)=\frac{1}{\kappa-1} \int_{x_{2}^{0}}^{x_{2}}\left(x_{2}^{-\kappa}-\xi^{-\kappa}\right) T_{1}(\xi) d \xi+c_{1} x_{2}^{1-\kappa}+c_{2} \quad \text { for } \quad \kappa \neq 1 \\
v_{30}^{I}\left(x_{2}\right)=\int_{x_{2}^{0}}^{x_{2}} T_{1}(\xi) \ln \frac{\xi}{x_{2}} d \xi+c_{1} \ln x_{2}+c_{2} \quad \text { for } \quad \kappa=1
\end{gathered}
$$

and

$$
v_{30}^{I I}\left(x_{2}\right)=\frac{x_{2}^{-\kappa}}{\kappa+1} \int_{x_{2}^{0}}^{x_{2}}\left(x_{2}^{\kappa+1}-\xi^{\kappa+1}\right) \xi^{-\kappa} T_{2}(\xi) d \xi+c_{1} x_{2}+c_{2} x_{2}^{-\kappa},
$$

respectively, where $\left.x_{2} \in\right] 0, L\left[\right.$ and $c_{\alpha}, \alpha=1,2$, are arbitrary constants.

Under some restriction on $T_{\alpha}\left(x_{2}\right), \quad \alpha=1,2$, the integral summands in (74) and (75) become continuous on [0,L]. Solutions (74)-(76) can always satisfy the boundary condition

$$
v_{30}(L)=v_{30}^{L}=\text { const }
$$

at the non-cusped edge $x_{2}=L$. Taking into account (77), from (74)-(76) it follows that either

$$
\begin{gathered}
c_{1}=L^{\kappa-1}\left[v_{30}^{L}-c_{2}-\frac{1}{\kappa-1} \int_{x_{2}^{0}}^{L}\left(L^{-\kappa}-\xi^{-\kappa}\right) T_{1}(\xi) d \xi\right] \text { for } 0 \leq \kappa<1 \text { and for } \kappa>1, \\
c_{1}=\ln ^{-1} L\left[v_{30}^{L}-c_{2}-\int_{x_{2}^{0}}^{L} T_{1}(\xi) \ln \frac{\xi}{L} d \xi\right] \text { for } \kappa=1,
\end{gathered}
$$

and

$$
c_{1}=L^{-1}\left[v_{30}^{L}-c_{2} L^{-\kappa}-\frac{L^{-\kappa}}{\kappa+1} \int_{x_{2}^{0}}^{L}\left(L^{\kappa+1}-\xi^{\kappa+1}\right) T_{2}(\xi) d \xi\right] \text { for } \kappa \geq 0
$$

respectively, or

$$
\begin{gathered}
c_{2}=v_{30}^{L}-c_{1} L^{1-\kappa}-\frac{1}{\kappa-1} \int_{x_{2}^{0}}^{L}\left(L^{-\kappa}-\xi^{-\kappa}\right) T_{1}(\xi) d \xi \text { for } 0 \leq \kappa<1 \text { and for } \kappa>1, \\
c_{2}=v_{30}^{L}-c_{1} L^{1-\kappa}-\int_{x_{2}^{0}}^{L} T_{1}(\xi) \ln \frac{\xi}{L} d \xi \text { for } \kappa=1,
\end{gathered}
$$

and

$$
c_{2}=L^{\kappa}\left[v_{30}^{L}-c_{1} L-\frac{L^{-\kappa}}{\kappa+1} \int_{x_{2}^{0}}^{L}\left(L^{\kappa+1}-\xi^{\kappa+1}\right) T_{2}(\xi) d \xi\right] \text { for } \kappa \geq 0,
$$

respectively.

Now we try to satisfy the boundary condition

$$
v_{30}(0)=v_{17}^{0}=\text { const }
$$


at the cusped edge $x_{2}=0$. As is obvious, (74)-(76) are unbounded as $x_{2} \rightarrow 0+$ by $\kappa>1, \kappa=1$ and $\kappa>0$, respectively, and in these cases (84) can not be fulfilled. For boundedness of solutions we have to take $c_{1}=0$ and $c_{2}=0$ for (74), (75) and (76), respectively. So, Keldysh type problem can be well-posed.

Unique bounded solutions $v_{30} \in C^{2}(] 0, L[)$ of the Keldysh type problems (72), (77) with $\kappa \geq 1$ and (73), (77) with $\kappa>0$, by virtue of (78)-(80) (or (81)-(83)), have the forms $\left(x_{2}^{0}=0\right)$

$$
v_{30}^{I}\left(x_{2}\right)=\frac{1}{\kappa-1} \int_{0}^{x_{2}}\left(x_{2}^{-\kappa}-\xi^{-\kappa}\right) T_{1}(\xi) d \xi+v_{30}^{L}-\frac{1}{\kappa-1} \int_{0}^{L}\left(L^{-\kappa}-\xi^{-\kappa}\right) T_{1}(\xi) d \xi, \quad \kappa>1
$$

(provided $T_{1}\left(x_{2}\right)$ is integrable on $] 0, L\left[\right.$ and $T_{1}\left(x_{2}\right)=O\left(x_{2}^{\delta}\right), \quad \delta>\kappa-1, x_{2} \rightarrow 0+$ ),

$$
v_{30}^{I}\left(x_{2}\right)=\int_{0}^{x_{2}} T_{1}(\xi) \ln \frac{\xi}{x_{2}} d \xi+v_{30}^{L}-\int_{0}^{L} T_{1}(\xi) \ln \frac{\xi}{L} d \xi, \quad \kappa=1
$$

(provided $T_{1}\left(x_{2}\right)$ is integrable and bounded on $] 0, L[$ ) and

$$
v_{30}^{I I}\left(x_{2}\right)=\frac{x_{2}^{-\kappa}}{\kappa+1} \int_{x_{2}^{0}}^{x_{2}}\left(x_{2}^{\kappa+1}-\xi^{\kappa+1}\right) \xi^{-\kappa} T_{2}(\xi) d \xi+L^{-1}\left[v_{30}^{L}-\frac{L^{-\kappa}}{\kappa+1} \int_{x_{2}^{0}}^{L}\left(L^{\kappa+1}-\xi^{\kappa+1}\right) T_{2}(\xi) d \xi\right] x_{2}, \quad \kappa>0
$$

(provided $T_{2}(\xi)$ is integrable on $] 0, L[$ and for $\kappa \geq 2$

$$
\left.T_{2}\left(x_{2}\right)=O\left(x_{2}^{\delta}\right), \quad \delta>\kappa-2, \quad x_{2} \rightarrow 0+\right) .
$$

Solutions (74) and (76) can fulfil (84) if correspondingly $0 \leq \kappa<1$ and $\kappa=0$. In these cases from (84) there follows that $\left(x_{2}^{0}=0\right)$

$$
c_{2}=v_{30}^{0},
$$

provided $T_{\alpha}\left(x_{2}\right), \quad \alpha=1,2$, are integrable on $[0, L]$.

Thus, unique solutions $v_{30} \in C^{2}(] 0, L[) \cap C([0, L])$ of the Dirichlet type problems (72), (77), (84) with $0 \leq \kappa<1$ and (73), (77), (84) with $\kappa=0$, by virtue of (78), (80) (or (81), (83)), have the forms

$$
\begin{aligned}
& v_{30}^{I}\left(x_{2}\right)=\frac{1}{\kappa-1} \int_{0}^{x_{2}}\left(x_{2}^{-\kappa}-\xi^{-\kappa}\right) T_{1}(\xi) d \xi \\
& +L^{\kappa-1}\left[v_{30}^{L}-v_{30}^{0}-\frac{1}{\kappa-1} \int_{0}^{L}\left(L^{-\kappa}-\xi^{-\kappa}\right) T_{1}(\xi) d \xi\right] x_{2}^{1-\kappa}+v_{30}^{0}, \quad 0 \leq \kappa<1,
\end{aligned}
$$

and

$$
v_{30}^{I I}\left(x_{2}\right)=\int_{0}^{x_{2}}\left(x_{2}-\xi\right) T_{2}(\xi) d \xi+L^{-1}\left[v_{30}^{L}-v_{30}^{0}-\int_{0}^{L}(L-\xi) T_{2}(\xi) d \xi\right] x_{2}+v_{30}^{0}
$$

respectively.

So, we arrive at the following conclusion:

(i) If $0 \leq \kappa<1$, solution (74) is continuous on [0,L], therefore boundary conditions can be satisfied on both the non-cusped $\left(x_{2}=L\right)$ and cusped $\left(x_{2}=0\right)$ edges and by means of $(74)$ a unique solution of the Dirichlet type boundary value problem can be written in the explicit form;

(ii) If $\kappa \geq 1$, taking $c_{1}=0$ we avoid unboundedness of the solutions (74), (75) as $x_{2} \rightarrow 0+$ and, in view of (75), a unique solution can be constructed by means of a boundary condition at the non-cusped edge (Keldysh type boundary value problem); 
(iii) For any $k>0$ solution (76) is unbounded as $x_{2} \rightarrow 0+$ unless $c_{2}=0$ and if $c_{2}=0$, by virtue of (76), we can construct in the explicit form a unique solution of the Keldysh problem with a prescribed value at the non-cusped edge $\left(x_{2}=L\right)$;

(iv) Unbounded solutions of Keldysh type problems (72), (77) and (73), (77) are defined up to the summand $c_{1} x_{2}^{1-k}$ for $\kappa>1\left(c_{1} \ln x_{2}\right.$ for $\left.\kappa=1\right)$ and $c_{2} x_{2}^{-\kappa}$, respectively, where $c_{\alpha}, \alpha=1,2$, are arbitrary constants.

From conclusions (i)-(iii) it is clear that the presence of a cusped edge depending, on sharpening geometry at a cusped edge, causes change of the Dirichlet type problem by the Keldysh type problem for ensuring well-posedeness of boundary value problems in displacements. Moveover, in contrast to Model I, where depending on sharpening geometry of the cusped edge (in other words of the kind of degeneration of equations under consideration) arise both the Dirichlet and Keldysh type problems, in Model II presence of cusped edges always demands consideration of the Keldysh type problem.

From (74), (75) it is easily seen that under obvious restrictions on $T_{1}\left(x_{2}\right)$ the boundary value problem, when on the non-cusped edge boundary conditon (77) and on the cusped edge boundary conditon (see (40))

$$
X_{320}(0)=\lim _{x_{2} \rightarrow 0+} \mu h v_{30,2}=X_{0}=\text { const }
$$

are prescribed, is correct. The solution $v_{30}^{I}$ can be represented in the explicit form. If on the non-cusped edge we set boundary conditon

$$
X_{320}(L)=X_{L}=\text { const },
$$

then a solution $v_{30}^{I}$ will be determined up to the additive constant. If $0 \leq \kappa<1$, the last constant can be uniquely determined by boundary condition (84). Hence, the last boundary value problem will be correct.

From (76) it is easily seen that under obvious restrictions on $T_{2}\left(x_{2}\right)$ the boundary value problem, when on the non-cusped edge boundary condition (see (40))

$$
X_{320}(L)=\mu\left[\frac{h_{0}}{2}\left(x_{2}^{k} v_{30}\right),\left.2\right|_{x_{2}=L}+u_{3}\left(x_{1}, L, \stackrel{(-)}{h} L_{0}^{K}\right) \kappa \stackrel{(-)}{h} L_{0}^{\kappa-1}-u_{3}\left(x_{1}, L, \stackrel{(+)}{h_{0}} L^{\kappa}\right) \kappa h_{0} L^{\kappa-1}\right]=X_{L}
$$

is prescribed, a solution $v_{30}^{I I}$ can be determined up to the addend $c_{2} x_{2}^{-\kappa}$ and is unbounded unless $c_{2}=0$.

$$
X_{320}(0)=\mu \lim _{x_{2} \rightarrow 0+}\left\{\frac{h_{0}}{2} x_{2}^{\kappa} \int_{x_{2}^{0}}^{x_{2}} \xi^{-\kappa} T_{2}(\xi) d \xi+\left[u_{3}\left(x_{1}, x_{2}, \stackrel{(-)}{h_{0}} x_{2}^{\kappa}\right) \stackrel{(-)}{h_{0}}-u_{3}\left(x_{1}, x_{2}, \stackrel{(+)}{h_{0}} x_{2}^{\kappa}\right) \stackrel{(+)}{h}_{0}\right] \kappa x_{2}^{\kappa-1}\right\}
$$

and can never be prescribed arbitrarily, i.e. in Model II cusped edges should be released from boundary conditions.

\section{Some Examples of Application to Composite Structures}

If we consider Model III for an orthotropic non-homogeneous material in the static case under the assumptions $u_{\alpha} \equiv 0$, i.e., $v_{\alpha 0} \equiv 0, \quad \alpha=1,2$, (71), from (33) we obtain only one governing equation

$$
\begin{aligned}
& \frac{h_{0}}{4}\left[\left(E_{3131} x_{2}^{k} v_{30,1}\right)_{1}+\left(E_{3232} x_{2}^{K} v_{30,2}\right), 2\right]+Q_{v_{3}}\left(x_{1}, x_{2}\right) \sqrt{\kappa^{2} h_{0}^{(+)} x_{2}^{2(\kappa-1)}+1} \\
& +Q_{(-))}\left(x_{1}, x_{2}\right) \sqrt{\kappa^{(-)} h_{0}^{2} x_{2}^{2(k-1)}+1}+\Phi_{30}=0 \quad \text { in } \omega .
\end{aligned}
$$

For the composite structure we take the effective moduli

$$
\begin{aligned}
& E_{3131}=E_{3131}^{f} v_{f}+E_{3131}^{m}\left(1-v_{f}\right), \\
& E_{3232}=E_{3232}^{f} v_{f}+E_{3232}^{m}\left(1-v_{f}\right)
\end{aligned}
$$

and the effective volume force

$$
\Phi_{30}=\Phi_{30}^{f} v_{f}+\Phi_{30}^{m}\left(1-v_{f}\right)
$$


where $v_{f}$ is the fiber volume fraction. The indices $f$ and $m$ denote fiber and matrix, respectively.

If $E_{3131}=$ const,$\quad E_{3232}=$ const, then from (85) we obtain

$$
\begin{aligned}
& \frac{E_{3131}}{E_{3232}} x_{2} v_{30,11}+x_{2} v_{30,22}+\kappa v_{30,2} \\
& +\frac{4 x_{2}^{1-\kappa}}{h_{0} E_{3232}}\left[Q_{(+)}\left(x_{1}, x_{2}\right) \sqrt{\kappa^{2} h_{0}^{2} x_{2}^{2(\kappa-1)}+1}\right. \\
& \left.+Q_{v 3}\left(x_{1}, x_{2}\right) \sqrt{\kappa^{2} h_{0}^{(-)} x_{2}^{2(\kappa-1)}+1}+\Phi_{30}\right]=0
\end{aligned}
$$

Introducing $x_{1}^{*}$ by $x_{1}=\left(\frac{E_{3131}}{E_{3232}}\right)^{\frac{1}{2}} x_{1}^{*}$ equation (86) we rewrite as

$$
\begin{aligned}
& x_{2}\left(\frac{\partial^{2} v_{30}}{\partial x_{1}^{* 2}}+\frac{\partial^{2} v_{30}}{\partial x_{2}^{2}}\right)+\kappa v_{30,2} \\
& +\frac{4 x_{2}^{1-\kappa}}{h_{0} E_{3232}}\left[Q_{(+)}\left(x_{1}, x_{2}\right) \sqrt{\kappa^{2} h_{0}^{2} x_{2}^{2(\kappa-1)}+1}\right. \\
& \left.+Q_{(-)}\left(x_{1}, x_{2}\right) \sqrt{\kappa^{2} h_{0}^{(-)} x_{2}^{2(\kappa-1)}+1}+\Phi_{30}\left(x_{1}, x_{2}\right)\right]=0
\end{aligned}
$$

and, by virtue of Theorem 2, we conclude that if $0 \leq \kappa<1$, at the edge $x_{2}=0$, the unknown $v_{30}$ can be prescribed, while for $\kappa \geq 1$ the boundary condition should be replaced by the boundedness of $v_{30}$ near the edge $x_{2}=0$.

So, if $0 \leq \kappa<1$, for the well-posedness of boundary value problem $v_{30}$ should be prescribed on the whole boundary of the body projection on the plane $x_{3}=0$ (Dirichlet problem is well-posed); if $\kappa \geq 1$ for the well-posedness of the boundary value problem $v_{30}$ should be prescribed only on the non-cusped part of the above-mentioned boundary (Keldysh problem is well-posed).

If in particular $v_{30}$ depends only on $x_{2}$, then from (86) we obtain

$$
\begin{aligned}
& \left(x_{1}^{\kappa} v_{30,2}\right)_{, 2}+\frac{4}{h_{0} E_{3232}}\left[Q_{(+)}\left(x_{2}\right) \sqrt{\kappa^{2} h_{0}^{2} x_{2}^{2(\kappa-1)}+1}\right. \\
& \left.+Q_{(-))}\left(x_{2}\right) \sqrt{\kappa^{2} h_{0}^{2} x_{2}^{2(\kappa-1)}+1}+\Phi_{30}\left(x_{2}\right)\right]=0
\end{aligned}
$$

Equation (87) is similar to equation (72) (mathematically they are the same) and its general solution has the form similar to (74), (75):

$$
\begin{aligned}
& v_{30}\left(x_{2}\right)=\frac{4}{(\kappa-1) h_{0} E_{3232}} \int_{x_{2}^{0}}^{x_{2}}\left(x_{2}^{-\kappa}-\xi^{-\kappa}\right)\left[Q_{v 3}^{(+)}(\xi) \sqrt{\kappa^{2} h_{0}^{2} \xi^{2(\kappa-1)}+1}\right. \\
& \left.+Q_{(-)}(\xi) \sqrt{\kappa^{2} h_{0}^{2} \xi^{2(\kappa-1)}+1}+\Phi_{30}(\xi)\right] d \xi+c_{1} x_{2}^{1-\kappa}+c_{2} \quad \text { for } \kappa \neq 1, \\
& v_{30}\left(x_{2}\right)=\frac{4}{h_{0} E_{3232}} \int_{x_{2}^{0}}^{x_{2}}[\underbrace{Q_{(+)}}_{v 2}(\xi) \sqrt{\kappa^{2} h_{0}^{2}+1} \\
& \left.+Q_{(-)}^{(+)}(\xi) \sqrt{\kappa^{2} h_{0}^{(-)}+1}+\Phi_{30}(\xi)\right] \ln \frac{\xi}{x_{2}} d \xi+c_{1} \ln x_{2}+c_{2} \quad \text { for } \quad \kappa=1,
\end{aligned}
$$


which makes transparent the above-mentioned peculiarity of the well-posedness of the boundary value problem in displacements. The corresponding analysis coincides word for word with assertions carried out for (74) and (75) in Section 3.

If

$$
E_{3 \underline{\alpha} 3 \alpha}=E\left(x_{1}\right) E_{\underline{\alpha}} x_{2}^{K_{\alpha}}, \quad \alpha=1,2, \quad \text { in } \bar{\omega},
$$

where $E_{\alpha}=$ const $>0, \quad E\left(x_{1}\right) \geq c>0$ in $\bar{\omega}$, from (85) we arrive at the equation

$$
\begin{aligned}
& \frac{E_{1}}{E_{2}} x_{2}^{\kappa_{1}-\kappa_{2}+1} v_{30,11}+x_{2} v_{30,22}+\frac{E_{1}}{E_{2}}\left(\ln E\left(x_{1}\right)\right)_{1} x_{2}^{\kappa_{1}-\kappa_{2}+1} v_{30,1} \\
& +\left(\kappa+\kappa_{2}\right) v_{30,2}+\frac{4 x_{2}^{1-\kappa-\kappa_{2}}}{h_{0} E\left(x_{1}\right) E_{2}}\left[Q_{(+)}\left(x_{1}, x_{2}\right) \sqrt{\kappa^{2} h_{0}^{2} x_{2}^{(2(\kappa-1)}+1}\right. \\
& \left.+Q_{(-)}\left(x_{1}, x_{2}\right) \sqrt{\kappa^{2} h_{0}^{2} x_{2}^{2(\kappa-1)}+1}+\Phi_{30}\left(x_{1}, x_{2}\right)\right]=0 \text { in } \bar{\omega} .
\end{aligned}
$$

If $\kappa_{1}-\kappa_{2}+1 \geq 0$, since

$$
\frac{E_{1}}{E_{2}}\left(\ln E\left(x_{1}\right)\right)_{1} x_{2}^{\kappa_{1}-\kappa_{2}+1}=O\left(x_{2}^{\kappa_{1}-\kappa_{2}+1}\right), \quad x_{2} \rightarrow 0+,
$$

according to Theorem 2 for $\kappa+\kappa_{2}<1$ Dirichlet problem will be well-posed, while for $\kappa+\kappa_{2} \geq 1$ Keldysh problem will be well-posed.

We can consider tension-compression problems similarly assuming

$$
v_{\alpha 0} \equiv 0, \quad \alpha=1,2, \quad v_{30} \equiv 0 .
$$

In this case we will have to do with the systems (32) and (30) for orthotropic and general anisotropic materials, respectively, where $E_{\gamma \delta \alpha \beta}, E_{\gamma \delta 3 \beta}$, and $\Phi_{\alpha 0}$ should be replaced by the effective moduli

$$
\begin{gathered}
E_{\gamma \delta \alpha \beta}=E_{\gamma \delta \alpha \beta}^{f} v_{f}+E_{\gamma \delta \alpha \beta}^{m}\left(1-v_{f}\right), \\
E_{\gamma \delta 3 \beta}=E_{\gamma \delta 3 \beta}^{f} v_{f}+E_{\gamma \delta 3 \beta}^{m}\left(1-v_{f}\right)
\end{gathered}
$$

and the effective volume forces

$$
\Phi_{\alpha 0}=\Phi_{\alpha 0}^{f} v_{f}+\Phi_{\alpha 0}^{m}\left(1-v_{f}\right) .
$$

For bending problem we apply $N=(1,1,0)$ approximation. In this case

$$
\begin{gathered}
u_{\alpha}\left(x_{1}, x_{2}, x_{3}, t\right)=\frac{1}{2} v_{\alpha 0}\left(x_{1}, x_{2}, t\right)+\frac{3}{2} v_{\alpha 1}\left(x_{1}, x_{2}, t\right)\left[x_{3}-\frac{\stackrel{(+)}{h}\left(x_{1}, x_{2}\right)+\stackrel{(-)}{h}\left(x_{1}, x_{2}\right)}{2}\right], \alpha=1,2 ; \\
u_{3}\left(x_{1}, x_{2}, x_{3}, t\right)=\frac{1}{2} v_{30}\left(x_{1}, x_{2}, t\right) .
\end{gathered}
$$

\section{Conclusions}

1) Hierarchical models for cusped, in general, elastic prismatic shells under mixed conditions on the face surfaces are constructed in both the cases of isotropic and non-homogeneous anisotropic, in particular, orthotropic materials. Such models are important in applications, e.g., in the study of seismic processes [28].

2) In models I-IV equations charachterizing tension-compression (see, correspondingly, systems (63), (65), (24), and (42)) and bending (more precisly deflection in $N=0$ approximation (see, correspondingly, equations (64), (66), (25), and (43)) can be considered separatly. This is not the fact in the case of models V-VIII.

3) Setting boundary conditions at edges in displacements depends on what is considered to be known on the face surfaces, stresses or displacements; moreover, assuming as known on the face surfaces at least one of the stress vector components, it will depend on the sharpening geometry of the cusped edge. The criteria, when the boundary 
conditions are classical (Dirichlet type) or nonclassical (Keldysh type), are established (compare with results in [3]). If on both the face surfaces only displacements are assumed as known, at cusped edges neither displacement nor integrated stress vectors can be prescribed. In other words, cusped edge should be released from boundary conditions.

4) In the static case, in the $N=0$ approximation, when the cusped prismatic shell-like body projection is a halfplane $x_{2} \geq 0$, the thickness $2 h=h_{0} x_{2}^{k}, \quad h_{0}=$ const $>0, \quad \kappa=$ const $\geq 0$, and at the edge $x_{2}=0$ integrated stress vector (either distributed but concentrated along the cusped edge or concentrated at point of the cusped edge load) is applied, the problem is solved in the explicit form (in quadratures) within the framework of Model IV. For $\kappa>0$ prismatic shell-like body is cusped one; for $K=0$, in particular, we have plate of a constant thickness.

5) In order to illustrate an application of Vekua-type models to composite structures, as example, an unidirectional lamina with fibers parallel to $x_{2}$-axis under shear strain is considered. Tension-compression is treated as well.

Acknowledgment. The present paper has been fulfilled by financial support of the Shota Rustaveli National Science Foundation (Grants \# D-13/18 \& 30/28)

\section{References}

[1] Vekua I.N.: On One Method of Calculating of Prismatic Shells. Trudy Tbilis. Mat. Inst., 21:191-259 (1955) (Russian)

[2] Vekua I.N.: Shell Theory: General Methods of Construction. Pitman Advanced Publishing Program, Boston-London-Melbourne 1985

[3] Jaiani G.: Cusped Shell-like Structures. Springer, Heidelberg, Dordrecht, London, New York 2011

[4] Jaiani G.V.: On a Mathematical Model of Bars with Variable Rectangular Cross-Sections. ZAMM Z. Angew. Math. Mech., 81 (3):147-173 (2001)

[5] Reddy, J. N.: Mechanics of Laminated Composite Plates and Shells. Theory and Analysis, CRC Press, New York 2004

[6] Altenbach, H., Altenbach, J., Kissing. W.: Mechanics of Composite Structural Elements. Springer, Berlin, Heidelberg, New york, 2004

[7] Mallikarguwa, T. Kant: A critical Review and some results of recently developed refined theories of fiber-reinforced laminated composites and sandwiches. Composite Structures, 23, 293-312 (1993)

[8] Carrera, E., Brischetto S.: Analysis of thickness locking in classical, refined, and mixed multilayered plate theories. Composite Structures, $82(4), 549-562(2008)$

[9] Carrera, E., Brischetto, S.: Analysis of thickness locking in classical, refined, and mixed multilayered plate theories. Composite Structures, 85(1), 83-90 (2008)

[10] Reddy, J. N.: An evaluation of equivalent-single-layer and layer theories of composite laminates. Composite Structures, 25, 21-35 (1993)

[11] Meunargia, T.: On construction of approximate solutions of equations of nonlinear and nonshallow shells. Journal of Mathematical Sciences, 157(1), 98-118 (2009)

[12] Vashakmadze, T.S.: The Theory of Anisotropic Elastic Plates (Kluwer Academic 1999). Springer-Science+Business Media B.V., Reprint, 2010

[13] Gordeziani, D., Avalishvili, G., Avalishvili, M.: Hierarchical models of elastic shells in Curvilinear coordinates. An International Journal Computers \& Mathematics with Applications, 51, 1789-1808 (2006)

[14] Jaiani, G., Kharibegashvili, S., Natroshvili, D., Wendland, W.L.: Hierarchical models for elastic cusped plates and beams. Lect. Notes TICMI 4 (2003)

[15] Jaiani, G., Kharibegashvili, S., Natroshvili, D., Wendland, W.L.: Two-dimensional hierarchical models for prismatic shells with thickness vanishing at the boundary. J. Elasticity 77(2), 95-122 (2004)

[16] Jaiani, G.: On a physical interpretation of Fichera's function. Acad. Naz. dei Lincei, Rend. della Sc. Fis. Mat. e Nat. S. 8, 68, fasc. 5, 426-435 (1980)

[17] Jaiani, G.V.: The first boundary value problem of cusped prismatic shell theory in zero approximation of Vekua's theory. Proc. I. Vekua Inst. Appl. Math. 29, 5-38 (1988) (Russian)

[18] Jaiani, G.: Application of Vekua's dimension reduction method to cusped plates and bars. Bull. TICMI 5, 27-34 (2001)

[19] Chinchaladze, N., Gilbert, R., Jaiani, G., Kharibegashvili, S., Natroshvili, D.: Existence and uniqueness theorems for cusped prismatic shells in the N-th hierarchical model. Math. Methods Appl. Sci. 31(11), 1345-1367 (2008)

[20] Jaiani, G.: A cusped prismatic shell-like body with the angular projection under the action of a concentrated force. Rendiconti Academia Nazionale delle Scienze detta dei XL Memorie di Matematica e Applicazioni, $124^{0}$, XXX, facs. 1, 65-82 (2006)

[21] Jaiani, G.: A cusped prismatic shell-like Body under the action of concentrated moments. Z. Angew. Math. Phys. 59, 518-536 (2008)

[22] Jaiani, G.: On a nonlocal boundary value problem for a system of singular differential equations. Appl. Anal. 87(1), 83-97 (2008)

[23] Chinchaladze, N., Jaiani, G., Maistrenko, B., Podio-Guidugli, P.: Concentrated contact interactions in cuspidate prismatic shell-like bodies. Archive of Applied Mechanics, 81(10), 1487-1505 (2011)

[24] Jaiani, G.V.: On a wedge-shaped body arbitrary loaded along the cusped edge. Bull. Georgian Academy Sci, Vol.75, 2, pp. 309-312 (1974) (Russian with Georgian and English summaries)

[25] Jaiani, G.V.: On a generalization of the Keldysh theorem. Georgian Mathematical Journal, 2 (3), $291-297$ (1995)

[26] Keldysh, M.V.: On some cases of degeneration of an equation of elliptic type on the domain boundary, Dokl. Akad. Nauk SSSR, 77(2), 181-183 (1951)

[27] Bitsadze, A.V.: Equations of Mixed Type. Moscow, 1959 (Russian)

[28] Aghalovyan, L.: On Definition of Stress-Strain States of Earth Lithoshperic Plates and Possibilities for Prediction of Earthquakes. Book of Abstracts of II Annual Meeting of the Georgian Mechanical Union, December 15-17, 2011, Tbilisi, 2011 